\title{
L'homme et la mer aux Yasawa (îles Fidji)
}

Pêche, resorts et conservation de la nature dans un paradis touristique Man and the sea in the Yasawa. Fishing, resorts and nature conservation in a tourist paradise (Fiji Islands)

\section{Émilie Nolet}

\section{OpenEdition Journals}

Édition électronique

URL : http://journals.openedition.org/ethnoecologie/3805

DOI : 10.4000/ethnoecologie.3805

ISSN : 2267-2419

\section{Éditeur}

Laboratoire Eco-anthropologie et Ethnobiologie

\section{Référence électronique}

Émilie Nolet, «L'homme et la mer aux Yasawa (îles Fidji) », Revue d'ethnoécologie [En ligne], 14 | 2018,

mis en ligne le 31 décembre 2018, consulté le 01 mai 2019. URL : http://journals.openedition.org/ ethnoecologie/3805; DOI : 10.4000/ethnoecologie.3805

Ce document a été généré automatiquement le 1 mai 2019.

\section{(1) $\$$}

Revue d'ethnoécologie est mis à disposition selon les termes de la licence Creative Commons Attribution - Pas d'Utilisation Commerciale - Pas de Modification 4.0 International. 


\section{L'homme et la mer aux Yasawa (îles Fidji)}

Pêche, resorts et conservation de la nature dans un paradis touristique

Man and the sea in the Yasawa. Fishing, resorts and nature conservation in a tourist paradise (Fiji Islands)

\section{Émilie Nolet}

\section{Introduction}

1 La République de Fidji (aussi dite Fidji, ou îles Fidji) est un État insulaire du Pacifique Sud constitué de près de 330 îles, inclus à la Mélanésie mais généralement considéré comme une "zone frontière " entre cette aire culturelle et celle de la Polynésie. Le pays, qui comptait 884887 habitants en 2017, a été rattaché en 1874 à la Couronne britannique et fut l'un des premiers d'Océanie à obtenir son indépendance en 1970. Si Fidji dut son essor économique à l'exploitation de la canne à sucre, son développement repose en large partie désormais sur le tourisme ${ }^{1}$, avec d'importantes différences à l'échelle des divers provinces et districts. Tandis que la côte orientale de Viti Levu (l'île principale, Figure 1) n'offre, en dehors de la capitale Suva, que peu d'infrastructures, une gamme pléthorique d'options est mise à la disposition des touristes sur la Côte de Corail du sud-ouest de l'île, à Nadi et dans les archipels des Mamanuca et Yasawa. Depuis les années 1960, le développement touristique a engendré de multiples transformations tant économiques que culturelles à Fidji (Pigliasco 2007, Connell 2015). Les revenus générés par les baux fonciers consentis aux grands groupes hôteliers installés dans le pays, les migrations induites par le travail salarié au sein des resorts (complexes hôteliers proposant des activités de loisir) ou une moindre dépendance à l'égard des ressources agricoles et halieutiques contribuent à redistribuer les pouvoirs et les alliances, à recomposer les identités locales comme les modalités des rapports homme-nature. 
Figure 1 : Les Yasawa, un archipel dans l'archipel

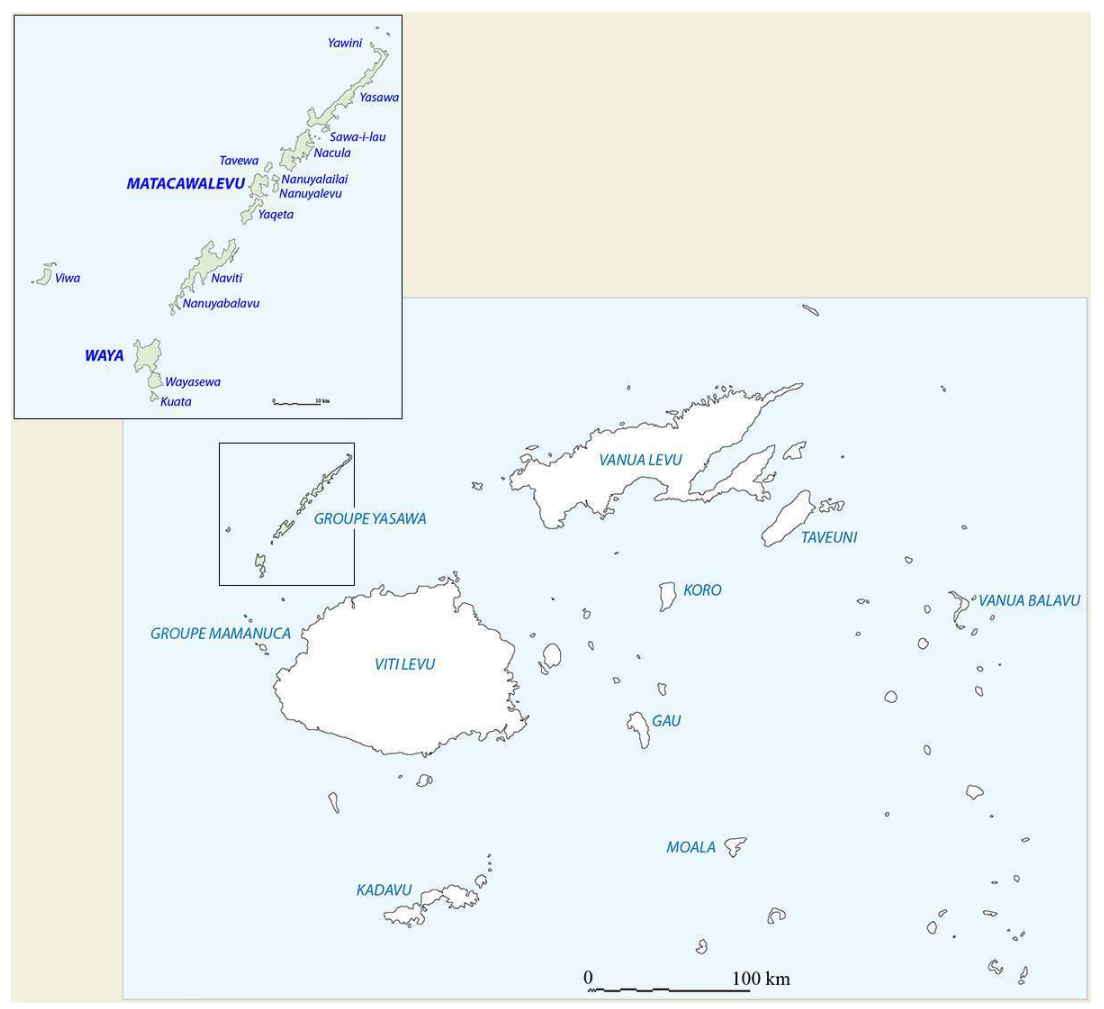

Carte de Fidji montrant la localisation des Yasawa

Réalisée par J. Suire, UMR 7041 ArScAn

Les Yasawa sont l'une des régions de Fidji qui bénéficient le plus du tourisme. Signalé pour la première fois en 1789 par W. Blight, l'archipel des Yasawa se compose d'une vingtaine d'îles volcaniques aujourd'hui associées à la province de $\mathrm{Ba}$ et à la confédération de chefferies (matanitu) de Burebasaga. Cet ensemble insulaire situé au nord-ouest de Viti Levu possède peu de ressources terrestres commercialisables, mais a connu depuis une vingtaine d'années un fort essor économique. Réputées pour la beauté de leurs paysages rendus célèbres par deux adaptations cinématographiques du roman d'Henry De Vere Stacpoole The Blue Lagoon en 1949 et 1980, les Yasawa sont censées offrir la quintessence de l'exotisme insulaire recherché par les touristes internationaux. En deux décennies, le tourisme a développé l'emploi salarié, favorisé l'accès à de nouveaux biens de consommation et largement désenclavé cet archipel éloigné de l'île principale, dont les habitants subsistaient surtout grâce à la pêche ${ }^{2}$ et à la culture de jardins familiaux. L'importance des Yasawa en tant qu'image internationale de Fidji contraste avec le peu de connaissances accumulées sur les formes sociales et culturelles particulières à cet archipel, même si l'ouverture au tourisme a entraîné un regain d'intérêt scientifique. Des auteurs issus de divers champs disciplinaires ont ainsi analysé les spécificités de l'offre touristique locale (Connell 2015), l'entreprenariat en contexte villageois (Gibson 2012), l'expression de la tradition dans les spectacles culturels de resorts (Brutti 2018) ou les tensions et revendications ayant pu émerger avec les nouveaux enjeux financiers (Abramson 2004: 82-83). On ne dispose par contre que d'informations très limitées sur la manière dont le tourisme affecte les modes de représentation et d'usage du milieu naturel, et en particulier les modes d'appréhension du milieu marin. Par exemple, dans quelle mesure les activités de pêche peuvent-elles être délaissées au 
profit d'emplois salariés dans les resorts ou adaptées à des besoins socio-économiques nouveaux ? En quoi le tourisme peut-il contribuer à modifier les modes d'acquisition des produits marins ou encore à la valorisation d'espaces ou d'espèces spécifiques?

3 Si le tourisme est vu comme un atout pour l'économie fidjienne, il est aussi accusé de favoriser l'inondation de certaines zones côtières urbanisées ou la pollution marine. Depuis une trentaine d'années, des recherches ont révélé l'impact environnemental de la surpêche, de l'urbanisation, du tourisme, mais aussi de l'agriculture intensive et des activités industrielles au sein de ce pays considéré comme l'un des plus riches et développés de Mélanésie. D'après Teh et al. : "Many of Fiji's inshore reef fisheries are now threatened from over-fishing, destructive and illegal fishing, and pollution" (2009: 807; Cumming et al. 2002). La dénonciation des risques et dommages écologiques va de pair avec une attention scientifique et politique de plus en plus soutenue à l'égard du changement climatique, qui s'est renforcée en 2012 suite à une grave séries d'inondations, puis encore en 2016 avec le passage du cyclone Winston - responsable de dizaines de morts et de lourds dégâts matériels à Fidji.

4 Très impliqué dans les discussions internationales sur le climat, le gouvernement fidjien s'est positionné en porte-voix des petites nations d'Océanie confrontées aux conséquences d'une catastrophe planétaire à laquelle elles ne contribuent elles-mêmes que de façon très marginale. À l'intérieur du pays, le gouvernement et ses partenaires internationaux, le monde scientifique et les ONG diffusent aussi des recommandations de «bonnes pratiques» écologiques visant à mieux protéger le patrimoine naturel, à minimiser l'impact du changement climatique et/ou à améliorer le développement local. À côté de recherches visant à évaluer par exemple les capacités de résilience des récifs coralliens, ces préoccupations se traduisent par des programmes d'information et d'appui à la gestion des ressources, mettant souvent en avant la nécessité de placer les acteurs locaux au centre des processus de décision (approches de gestion dite concertée et participative) $)^{3}$. Des communautés sont ainsi incitées à redécouvrir des modes traditionnels de gestion des ressources ou à diversifier leurs activités pour limiter les pressions exercées sur le milieu. Ces approches largement relayées par les médias ne sont pas homogènes et émanent d'individus ou d'institutions dont les objectifs, les sources, les moyens et les bases idéologiques diffèrent. Elles représentent néanmoins, avec le constat empirique des dommages écologiques, un autre possible facteur de transformation des relations homme-nature, dont les appropriations et conséquences locales restent inégalement évaluées à Fidji.

5 Mon objectif sera d'examiner en quoi ces deux ensembles de phénomènes en partie liés le développement du tourisme et celui d'une préoccupation environnementale assortie d'appels à la vigilance et aux bonnes pratiques - peuvent contribuer à réagencer les modes d'usage et de représentation du monde marin, à partir de deux études de cas : celui du village de Yalobi (île de Waya Levu, ou Waya, Figure 2), et celui du village de Vuake (île de Matacawalevu) ${ }^{4}$, où des enquêtes anthropologiques ont été conduites en $2012^{5}$.

Dans une première partie, je montrerai que le tourisme peut entraîner la perte ou la mise à distance d'éléments du savoir coutumier, et en même temps favoriser l'émergence de nouvelles formes d'appréhension et d'usage du milieu marin. La présence des resorts encourage en particulier le développement d'une pêche commerciale qui représente un moyen alternatif d'ascension économique et sociale, tout en contribuant à élaborer et vitaliser des relations traditionnelles fortes entre individus et unités familiales. Nous verrons dans un second temps que les villageois de Vuake et Yalobi dénoncent à leur 
échelle des perturbations écologiques, mais que les chaînes de responsabilités envisagées (impact du tourisme, surpêche, changement climatique, etc.) et surtout les solutions préconisées ne sont pas forcément les mêmes que celles avancées par le gouvernement fidjien, le monde scientifique, les ONG. Il apparaîtra qu'une série de paramètres, comme les conditions d'accès au foncier ou la présence d'un ethos d'égalité villageoise influencent les modes d'interaction avec le monde marin et la perception des formes socialement acceptables d'action sur ce milieu.

Figure 2 : Yalobi, un village tourné vers la mer

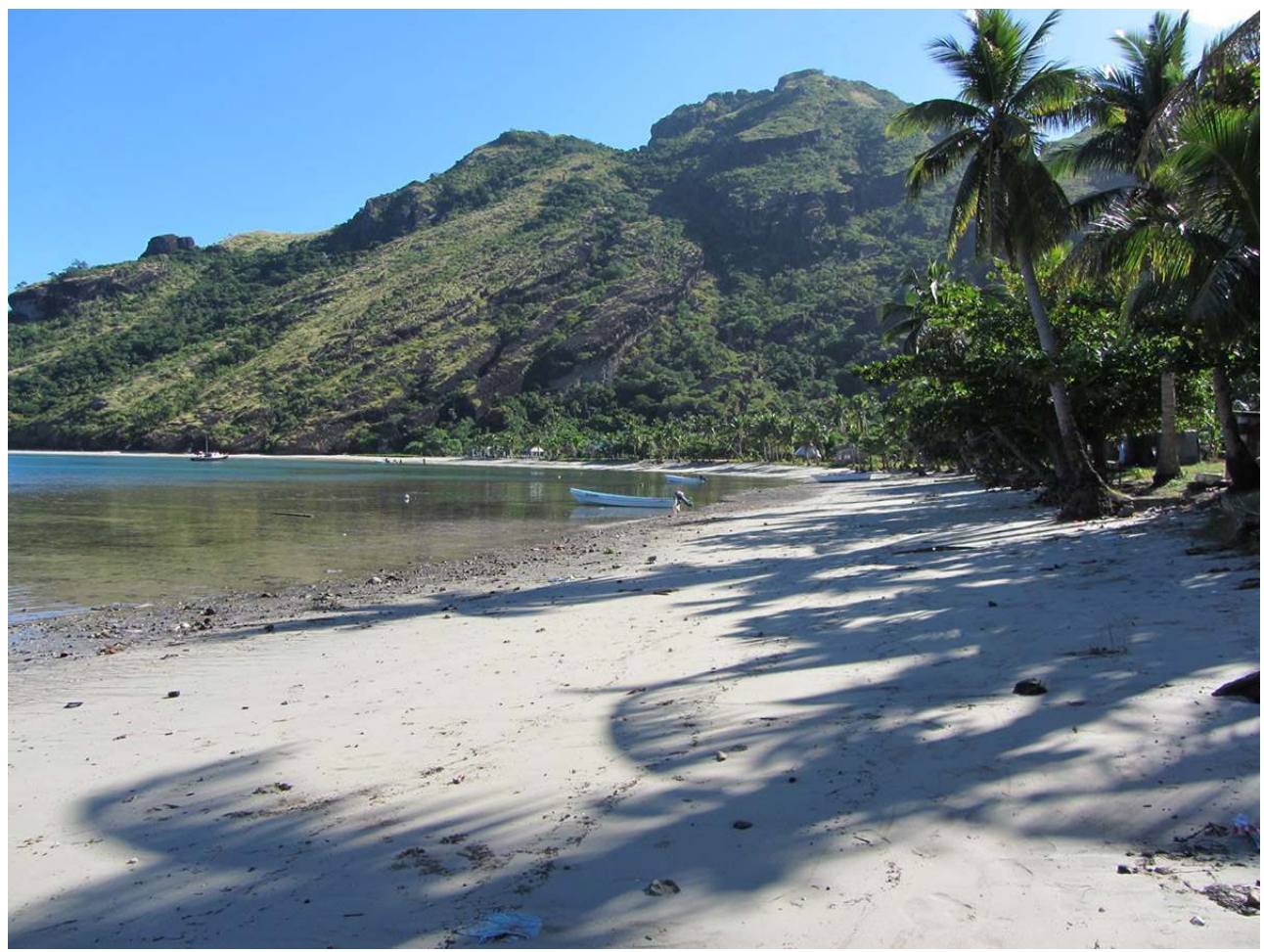

La plage de Yalobi, Waya

(c) É. Nolet 2012

\section{De la pêche au tourisme?}

\section{L'essor touristique : un phénomène récent}

7 Si les Yasawa sont aujourd'hui l'une des destinations les plus prisées de l'archipel fidjien, sans connaître encore les flux touristiques des Mamanuca, plus proches de Viti Levu, elles ne sont que récemment sorties de leur isolement. Au début des années 1940, les Yasawa avaient "peu de contact avec le reste de Fiji ", la population pratiquant une agriculture de subsistance complétée par la vente de coprah à Lautoka (Naval Intelligence Division 1944, cité par Connell 2015). Si le climat relativement sec et la proximité d'un aéroport international à Nadi sont d'évidents atouts, l'activité touristique s'est longtemps limitée au passage sporadique de navires de croisière, l'installation de nombreux resorts ${ }^{6}$, surtout sur les franges côtières, ne datant que des années 1990 (Connell 2015). L'archipel offre à présent une large gamme d'infrastructures, déclinant avec un luxe plus ou moins prononcé une offre touristique basée sur le farniente, les loisirs aquatiques, et des 
activités devant permettre une immersion culturelle à la fois modérée et fortement standardisée (Brutti 2018). Les resorts sont détenus en partie par des investisseurs étrangers, qui acquièrent des baux sur des terres détenues en indivision par les clans autochtones (native lands), et en partie par les communautés locales, où la majorité des employés sont recrutés (Figure 3). Les activités se déploient dans l'enceinte des resorts, dans l'espace marin immédiatement adjacent et sur des itinéraires soigneusement délimités dédiés à la plongée et au cabotage - même si les promenades impromptues des touristes et les visites guidées contribuent aussi à créer de nouvelles formes de circulation et de sociabilité dans les espaces intérieurs des îles.

8 Il est admis que pratiquement toutes les familles tirent des avantages matériels de la présence des touristes. Le salaire d'un employé de resort pourra être en partie redistribué dans sa proche parenté ou bien servir à l'achat de biens d'échange destinés aux cérémonies et compensations familiales. Outre les possibilités d'emploi salarié, mais rarement pérenne, dans les complexes hôteliers, les insulaires tirent des revenus de la vente d'artisanat lors des escales des bateaux de croisière ou encore organisent des visites payantes de sites naturels (Figure 4). La présence d'un resort sur une terre familiale est ainsi le plus souvent vue comme une excellente fortune : les revenus monétaires obtenus par l'emploi, la participation aux profits, l'emprunt, voir par des dons kerekere supposant une réciprocité différée (Toren 1999 : 151) permettent aux familles d'honorer leurs obligations coutumières (oga), de payer leurs frais scolaires, de financer les recharges pour téléphones portables, le kérosène utilisé comme combustible pour les fours de cuisine, le tabac ou le précieux kava (Piper methysticum). En regard, la plus grande partie des ressources alimentaires sera prélevée dans les jardins ou en mer, ou acquise par don et échange. Une activité touristique florissante apporte du prestige aux mataqali (clans) et aux chefs dont les terres génèrent des profits, l'émigration des jeunes est limitée et, dans les meilleurs cas, la qualité de vie - par exemple en termes d'accès à l'eau et au réseau électrique - peut être durablement améliorée. L'expansion du tourisme a été, aux Yasawa, un atout d'autant plus important que les conditions écologiques y sont assez peu favorables aux activités agricoles. L'exploitation des ressources terrestres reste en effet limitée par le manque d'espaces cultivables, la pauvreté des sols, la faible pluviométrie et l'éloignement de Viti Levu, qui impose de longs et couteux voyages pour la vente des produits de la terre. La beauté du vanua ${ }^{8}$ passe dans cette mesure pour un cadeau de Dieu et des ancêtres, et l'activité touristique, pour une manière acceptable de profiter de cet héritage.

9 La répartition géographique des resorts, qui investissent en priorité des plages spectaculaires, est cependant inégale et tous les insulaires ne bénéficient pas de la même façon des retombées du tourisme. Si certains ont la garantie d'une rente foncière et d'une source continue d'emploi local, d'autres dépendent de façon plus importante des ressources côtières et marines, prélevées dans ce que les Fidjiens nomment le qoliqoli ${ }^{9}$. Cette situation peut aussi être engendrée par des conflits fonciers empêchant le fonctionnement d'un resort communautaire, par des problèmes de gestion, par le recrutement prioritaire dans les mataqali dont les terres accueillent les resorts. Les habitants vont ainsi s'appuyer sur un ensemble de ressources, monétaires et non monétaires, qui interviennent dans des proportions variables selon les familles : revenus salariés du tourisme, produit des jardins (tovatova, où poussent ignames, bananes, manioc, etc.) et de la pêche, aides financières par des membres de la famille vivant en ville, petit commerce local de denrées non-périssables, cigarettes et kava. 
Figure 3 : Le resort, source d'emploi local

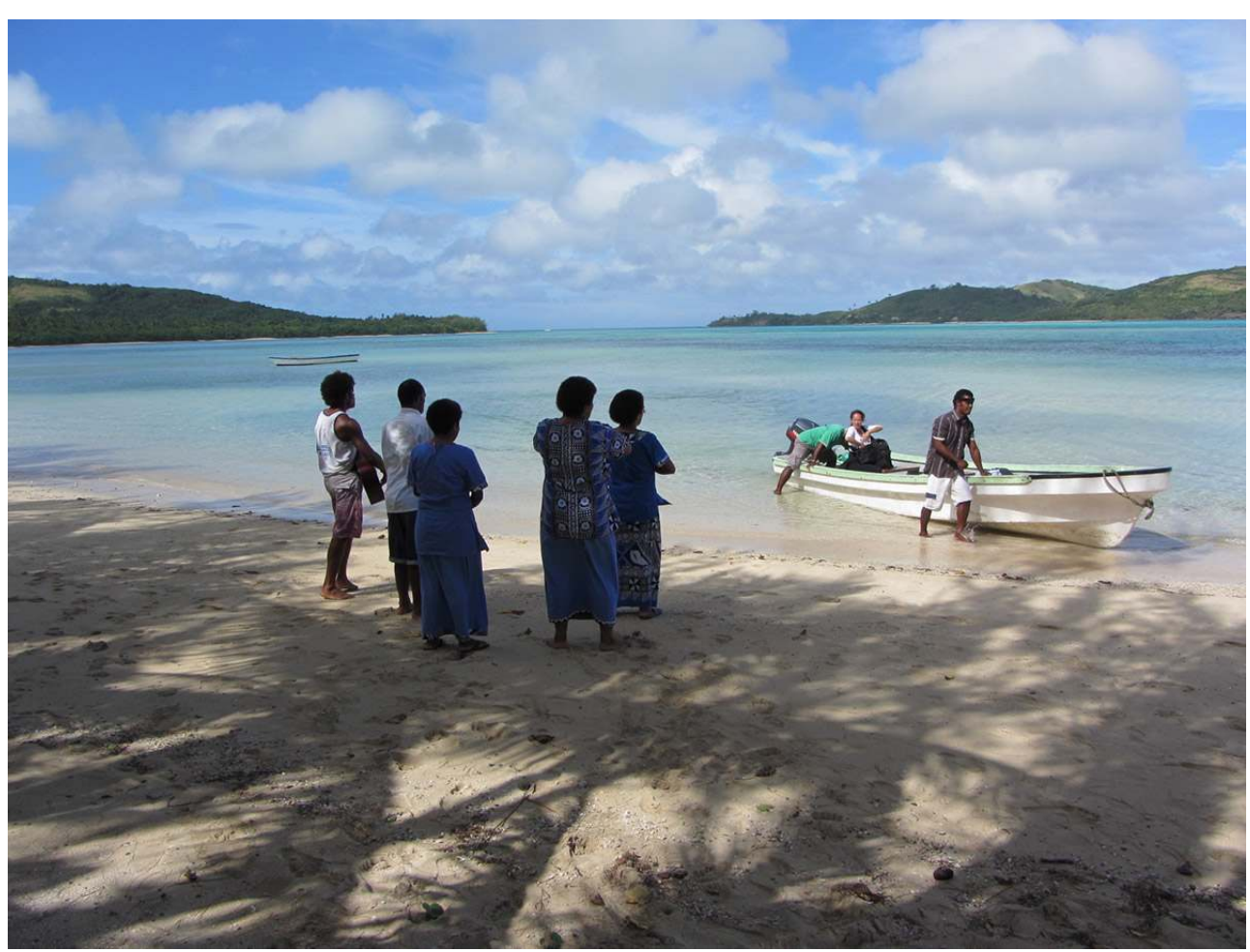

Employés d'un resort accueillant une visiteuse en chansons (c) É. Nolet 2012 
Figure 4 : De multiples manières de tirer parti du tourisme

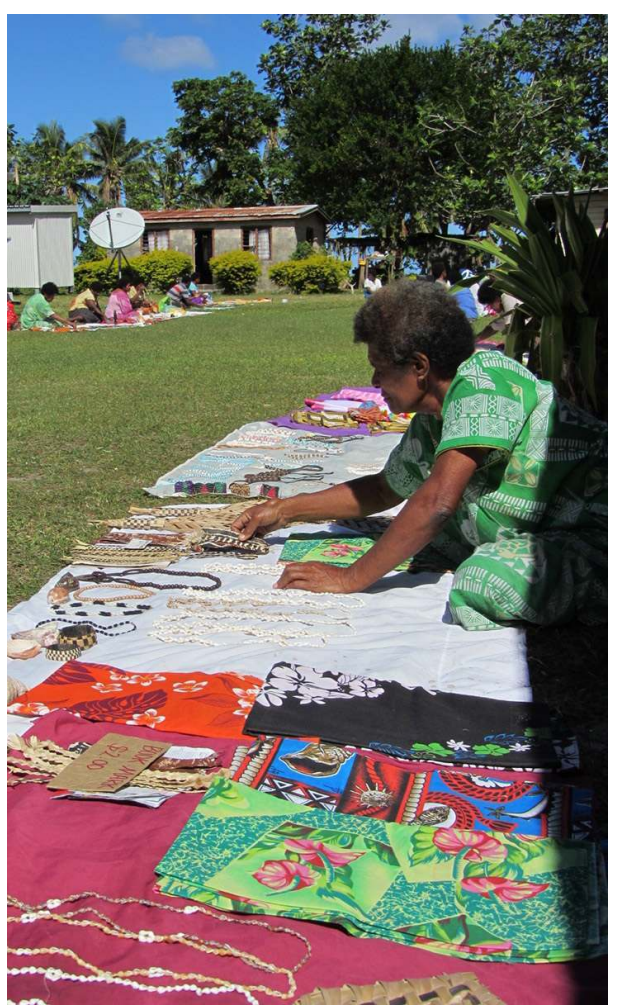

Femmes attendant le passage d'un bateau de croisière à Yalobi

(C) É. Nolet 2012

\section{De nouveaux types de rapports à la mer}

10 À Waya et Matacawalevu ${ }^{10}$, hommes, femmes et enfants participent tous aux activités de pêche et de collecte mais avec une assiduité variable, seul un petit nombre d'hommes étant vus comme des professionnels - en raison du temps passé en mer, de l'importance de leurs gains, et parce qu'on leur reconnaît savoir et compétence. Une partie des prises est destinée à la consommation et aux échanges avec les parents, amis, voisins et dignitaires du village ${ }^{11}$ :

« Maybe one, two, three of my neighbours here, if they don't go fishing, I send the fish to them. [...] If they bring fish too, they will share it to me, if I don't go fishing " (Figure 5).

11 Mais des commandes sont aussi désormais passées par les resorts à des pêcheurs souvent mieux équipés et plus aguerris que les autres, qui vont cibler des espèces ou catégories d'espèces particulières : des poissons de grande taille à la chair ferme et peu odorante, adaptés à la confection du kokoda (poisson cru mariné) apprécié des touristes, des bénitiers (vasua, Tridacna spp.) ou encore des langoustes (ura, Palinurus spp.) ensuite revendues jusqu'à $60 \mathrm{FJ}$ \$ pièce dans les restaurants. De la même manière, un pêcheur obtenant sans l'avoir forcément recherché un poisson de belle taille ou appartenant à une espèce valorisée entreprendra de le revendre à un resort voisin plutôt que de le consommer ou partager au village. Si les hommes réalisent la plus grande partie des pêches commerciales, des femmes s'investissent dans la vente de pieuvres (kuita, Octopus spp.), de crabes de boue (qari, scylla paramamosain) ou de coquillages très décoratifs 
comme les trocas (sici, Tectus niloticus) ou les davui (tritons géants, Charonia tritonis) aux resorts et aux touristes de passage.

L'essor du tourisme a ainsi ouvert de nouvelles opportunités, dans un climat de rivalité interfamiliale et interpersonnelle exacerbé : la proximité des resorts crée un nouveau marché pour les pêcheurs, et a l'avantage de limiter les coûts de transport et de réfrigération qui représentaient un frein à la commercialisation de produits marins à Viti Levu. En parallèle, des insulaires développent des micro-entreprises ciblant surtout là encore des espèces coûteuses, de grande taille - comme le thazard rayé, walu ( Scomberomorus commerson), vendu $11 \mathrm{FJ} \$$ le kilo en 2012, la carangue grosse tête, dole ( Caranx ignobilis), la saumonée léopard, donu (Plectropomus leopardus) -, les prises étant revendues à des grossistes à Lautoka ou écoulées sur les marchés de Viti Levu. On voit qu'un rapport marchand aux ressources marines tend à se renforcer, en partie à cause du tourisme, et contribue à éloigner certains taxons valorisés du circuit local des échanges et des prestations cérémonielles.

Figure 5 : Pêcher pour vivre et échanger

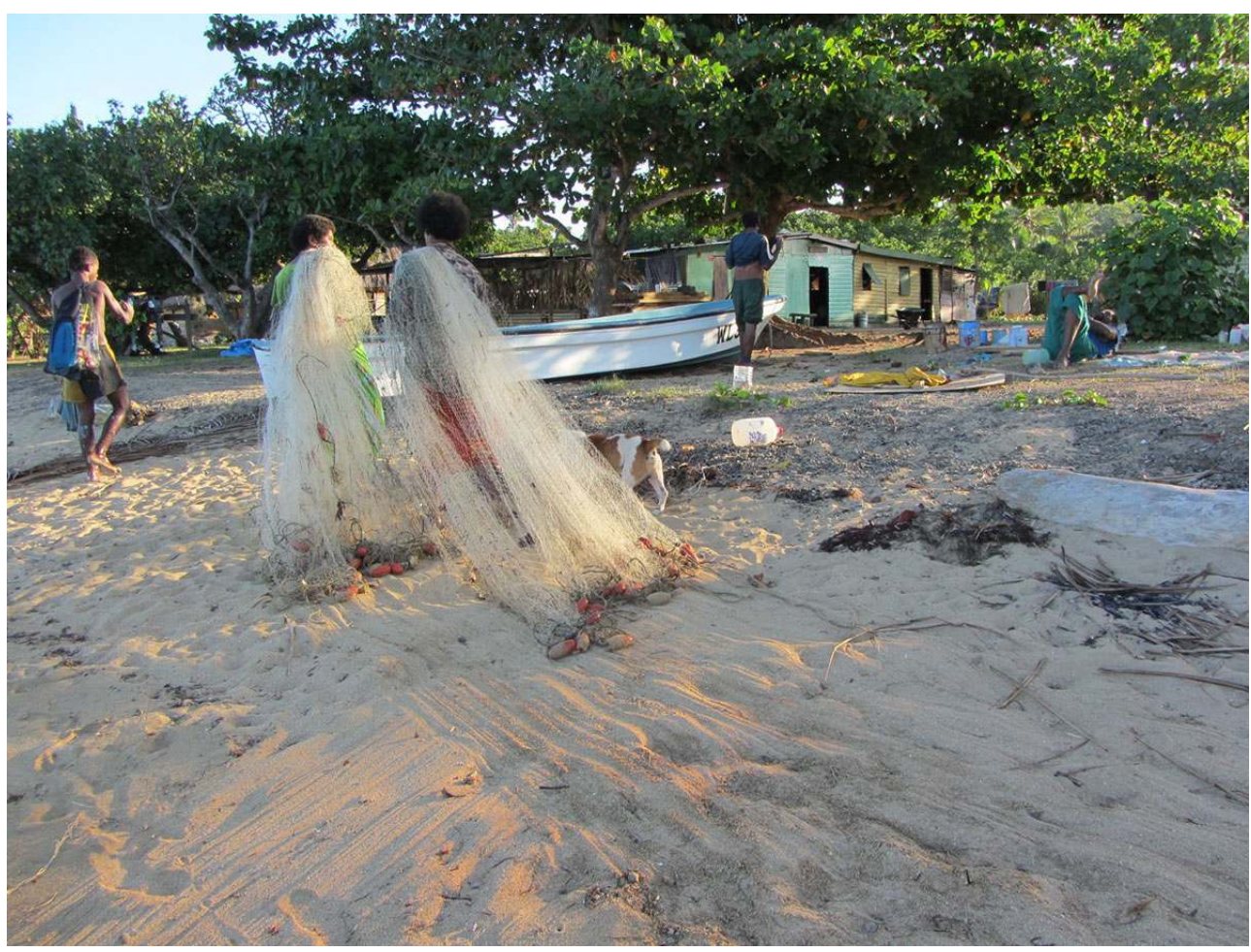

Femmes rentrant d'une expédition de pêche, sur la plage de Vuake

(c) É. Nolet 2012

Le développement du tourisme et celui d'une pêche commerciale encouragée par la proximité des resorts, l'élévation du coût de la vie et l'émergence d'un mode de pensée libéral valorisant l'entreprise individuelle, amènent ou accélèrent d'autres types de changements. Tout d'abord, les personnes employées régulièrement dans le secteur touristique ont moins de temps à consacrer à la pêche/collecte et recourent davantage à l'achat et au troc pour leurs besoins quotidiens ou les festins communautaires. L'éventail des techniques qu'elles mobilisent durant leur temps libre est souvent moins diversifié et prestigieux que celui des insulaires dont la pêche est l'activité principale (collecte sur la 
plage ou le platier récifal, pêche à la ligne en eau peu profonde, etc.) et axé sur des espèces communes, soit parce qu'elles «n'en maîtrisent pas » ou "plus » d'autres, soit parce qu'elles ont perdu tout intérêt pour des méthodes plus complexes ou spécialisées ce que les habitants qui n'ont pas accès aux emplois du secteur touristique interprètent comme une forme d'occidentalisation et une perte d'autonomie : "They don't know how to fish; they are fat. They buy from the shop » ironisait une pêcheuse de Vuake. Une ancienne employée de resort affirmait ainsi "ne pas savoir pêcher » et se cantonner à la collecte d'algues comme la caulerpe raisin, nama (Caulerpa racemosa) et de mollusques fouisseurs comme le mésodesme glabre, qeqe (Atactodea striata). Les insulaires qui n'ont jamais quitté le village tendent à employer un vocabulaire précis et spécialisé quand ils se réfèrent au monde marin, en faisant usage de termes locaux ${ }^{12}$, quand ceux qui ont longtemps travaillé dans les resorts peuvent se montrer plus vagues et privilégier les mots en dialecte de Bau ${ }^{13}$ (Pawley \& Sayaba 1971), l'idiome « national » des Mélanésiens de Fidji - en disant saqa au lieu de dole pour la carangue, par exemple.

Le tourisme n'est pas seul responsable de l'appauvrissement, de l'oubli ou d'une moindre utilisation de savoirs coutumiers relatifs à la mer, l'évolution plus générale des pratiques de consommation (plus grande disponibilité des conserves, de la viande importée) ou certaines croyances religieuses ${ }^{14}$ pouvant y participer. De plus, on constate qu'ils dépendent fortement du temps passé à l'extérieur du village et qu'ils concernent d'autres Fidjiens amenés à résider en milieu urbain, tels les militaires. Enfin, il n'est pas rare que d'anciens employés « réapprennent » la mer et le métier de pêcheur. Une femme ayant travaillé pendant des années comme cuisinière à Turtle Island Resort expliquait son bonheur d'être rentrée au village et vivait désormais principalement de la vente de crabes de boue et d'autres produits marins - continuant ainsi de produire des revenus monétaires, complétant les biens non monétaires générés par son époux dans la plantation familiale.

Il faut ajouter que si le tourisme peut entraîner une érosion des savoirs traditionnels, voire une distanciation à l'égard du milieu marin, il contribue aussi en parallèle à diversifier les modes d'exploitation de la mer: des villageois vont en effet devenir instructeurs et guides de plongée sous-marine aux abords de leurs îles, ou bien sont rémunérés pour des expéditions de pêche sportive ou de nourrissage des requins (shark feeding), destinées aux touristes aventureux. Les Fidjiens qui prennent en charge les touristes acquièrent des connaissances scientifiques sur l'écologie marine qu'ils ne possédaient en général pas auparavant (espèces menacées, biologie du corail, etc.), ou bien sont amenés à puiser dans un répertoire coutumier qu'ils n'auraient pas forcément mobilisé dans d'autres circonstances (nomination des différents types de coraux, identification des lieux d'agrégation d'espèces spectaculaires comme le Cheilinus undulatus ou Napoléon, etc.). En d'autres termes, d'autres formes de rapports à la mer sont explorées et d'autres champs de connaissance investis pour les besoins du tourisme. L'offre locale en matière de tourisme reflète d'ailleurs le désir, occidental ou autochtone, de faire converger des éléments issus de, ou inspirés par la coutume (initiation à la vannerie, visite de village...), des standards du tourisme international (contact avec des espèces marines de grande taille, recherche de sensations fortes) et des images exotiques de portée universelle, axées sur le monde marin et l'insularité (les visiteurs plongent au «Garden of Eden » à Naviti, sont logés au resort de la Pieuvre ou de la Raie Manta, etc.).

16 Autre phénomène important, on constate que la présence des resorts pousse les villageois à privilégier des méthodes de pêche très performantes et/ou adaptées aux espèces de 
grande taille, comme la pêche sous-marine utilisant un harpon muni d'un élastique ou un fusil - si efficace que sa pratique de nuit est comparée à un «meurtre »-, la pêche à la ligne en haute-mer, la pêche au filet (lawa ni ika). La pêche nocturne utilisant des torches ou générateurs (dont le bruit attirerait les walu) est très appréciée en raison de son efficacité (Gillett \& Moy 2006: 15, Veitayaki 1995: 13). Le choix d'une méthode dépendra des conditions extérieures (profondeur, calendrier lunaire, saison, etc.) des proies visées, de l'âge et de l'expérience des participants, qui mobiliseront lors d'une expédition un ensemble de savoirs coutumiers et « importés ».

17 Les revenus du tourisme permettent ainsi à davantage d'individus d'avoir accès à des équipements performants (fusils de fabrication australienne, glacières, torches de plongée, etc.), même si le nombre de bateaux supportant la haute-mer reste limité (3 pour 200 habitants à Vuake en 2012). Des techniques de pêche touchant des proies peu valorisées, faiblement efficaces, productives seulement une partie de l'année ou nécessitant la participation de nombreux insulaires sont tombées en désuétude (comme la pêche collective dite yavirau, employant un piège mobile en feuilles de cocotier, ou les pièges monumentaux dits taga à Waya), ou sont réservées aux besoins domestiques (harponnage de poissons en surface sur le récif par exemple) et à des ventes exceptionnelles (récolte à l'aide de moustiquaires en octobre-novembre des vers marins balolo, Eunice viridis, en partie consommés et en partie vendus à Viti Levu).

On ajoutera que le tourisme contribue à modifier les modes de circulation et l'appréhension de l'espace, à la fois sur terre et en mer. L'implantation des resorts a tout d'abord donné une importance et une valeur foncière inédites à des espaces insulaires à haut potentiel touristique. Des plages (baravi) autrefois dévolues aux activités de subsistance et fréquentées de manière occasionnelle sont devenues des pôles d'attraction et des enjeux de première importance. De même, des espaces marins qui n'étaient pas spécialement réputés pour leur intérêt économique ou leur beauté ont acquis une importance et une visibilité nouvelles avec la plongée de loisirs et ses "spots " parfois mondialement célèbres. Par ailleurs les digues, les espaces de baignade, les circuits des expéditions de plongée, les villages touristiques et autres restaurants de plage fabriquent de nouvelles frontières, de nouvelles routes, de nouveaux points d'ancrage et de repère géographiques, qui se surimposent aux références anciennes (toponymie, villages, fondations de maisons yavu, etc.) et même parfois les remplacent. Des espaces perçus comme sauvages dans l'intérieur des îles (ulu ni vanua) et la brousse (vekau) sont plus facilement traversés et investis par les activités humaines qu'autrefois. Ou bien des noms de resorts sont utilisés en lieu et place de la toponymie traditionnelle, par les personnels des complexes hôteliers ou la population en général.

Rappelons pour finir que si la pêche reste très pratiquée à Vuake et Yalobi, seule une poignée d'habitants est en mesure de vivre de la vente de produits marins de manière quasi-exclusive, les autres pratiquant une pêche plus occasionnelle, moins rentable, au milieu d'autres catégories de ressources et selon les opportunités du moment. Vivre pleinement de la pêche repose sur différents paramètres: le fait de posséder ou de pouvoir accéder par ses connexions familiales à un bateau (et donc aux zones de pêche en eau profonde, fréquentées par les plus gros poissons), de pouvoir mobiliser l'aide de ses parents et affins, d'avoir la santé et l'énergie suffisantes pour endurer les longues heures de veille, le froid, le voisinage des requins, entre en ligne de compte. La capacité à tisser des alliances plus ou moins pérennes avec des resorts, souvent par le biais de la parenté, est aussi impliquée. 
Dans tous les cas, le développement de la pêche marchande et du tourisme contribue à une professionnalisation des activités de pêche, avec un petit nombre de «spécialistes » tirant le meilleur parti du nouveau marché local, tandis qu'en parallèle une partie de la population s'éloigne du milieu marin ou se contente de ventes sporadiques. Comme l'expliquait un de ces specialistes :

« Now, we have plenty of opportunities around, with backpackers and resorts [...] When you're good on that, you still have plenty of fish. Because less people are doing fishing. That's one good thing the tourist industry brought to the islands » (I., 50 ans, Vuake).

\section{Les avantages du « business de la mer »}

21 La plupart des villageois perçoivent le tourisme comme une aubaine pour leur archipel excentré, régulièrement ravagé par les cyclones, et ne possédant ni le prestige coutumier, ni les ressources foncières, ni le poids politique des grandes chefferies de Viti Levu (Bose \& Fraenkel $2007: 230$ ). Certains ont pourtant aussi évoqué des conséquences négatives et des changements socio-culturels inquiétants. En plus de renforcer les inégalités dérivant des conditions d'accès au foncier, les resorts peuvent devenir une source de conflits : par exemple quand la logique entrepreneuriale s'exprime sans égards pour la parenté dans une structure communautaire, quand une branche familiale (y compris celle des chefs) capte une part trop massive des profits ou quand une concurrence commerciale, non résolue par l'entraide ou le partage de la clientèle, s'installe entre des clans. De même, la fréquentation des touristes et l'arrivée d'employés venus de Viti Levu sont tenues pour responsables d'une érosion accélérée des spécificités culturelles locales. Baignés de culture occidentale, les employés des resorts seraient moins enclins à respecter les tabous hiérarchiques et à s'impliquer dans les affaires du vanua. Les migrations professionnelles induites par le travail salarié auraient aussi tendance à distendre les réseaux familiaux et empêcheraient certains insulaires, dont des héritiers ou détenteurs de titres de chefferie, de jouer un rôle actif au sein de leurs villages. Des insulaires ont également pointé des risques de nature morale et en particulier des tentations d'adultère au sein des resorts espaces occidentalisés où les valeurs chrétiennes qui font partie intégrante de la «vie traditionnelle fidjienne », bula vakavanua, ne seraient selon eux pas aussi prégnantes qu'au village. Le fait de posséder une affaire rentable ou d'obtenir une place confortable dans un resort permet en outre à des individus ou familles d'acquérir un pouvoir d'influence parfois jugé peu conforme à leur position sociale (groupes seconds-venus par exemple). La présence des resorts peut ainsi être envisagée comme un élément perturbateur, travaillant contre un équilibre établi à la fois familial, culturel, religieux et politique. La reconnaissance de risques culturels liés au tourisme conduit d'ailleurs les insulaires à exiger que les étrangers en visite dans les villages respectent les usages et tabous coutumiers : genoux couverts, tête nue, offrande de kava à l'autorité locale, etc.

En regard, la pêche commerciale offrirait un certain nombre d'avantages, l'un des plus couramment cités étant de permettre à un individu de rester résider sur sa terre d'origine, avec les bénéfices matériels et sociopolitiques que ceci amène - comme la gratuité d'une large partie des ressources alimentaires, la possibilité de mobiliser l'aide du groupe parental ou d'être impliqué(e) dans la vie politique locale et de revendiquer plus aisément titres ou responsabilités. La pêche commerciale est aussi plus lucrative : un pêcheur ou une pêcheuse expérimenté(e) peut obtenir en quelques heures ce qu'il ou elle mettra une semaine à gagner en travaillant comme femme de chambre ou animateur 
(trice) dans un resort ${ }^{15}$. Des pêcheurs ont évoqué par ailleurs leur goût de l'indépendance et l'avantage de ne pas devoir s'insérer dans l'organisation très hiérarchisée d'un resort, en particulier si les supérieurs immédiats sont des « étrangers » occidentaux ou venus de Viti Levu ${ }^{16}$ (« free life, free time!»). Le fait de ne pas devoir attendre une ou deux semaines un modeste salaire, mais d'obtenir une somme d'argent immédiatement employable par la vente est encore un intérêt substantiel. De même, si bien vivre du tourisme implique souvent une position familiale favorable (être membre d'un mataqali dont les terres accueillent un resort, apparenté au chef, etc.), la pêche serait un domaine plus ouvert, laissant davantage de place aux compétences individuelles et à la chance.

On notera que la pêche, même lorsqu'elle s'exerce dans des conditions et avec des objectifs fort éloignés de la tradition, reste perçue comme une activité plus authentiquement "fidjienne » que le travail salarié, et qu'elle est à ce titre valorisée. Pêcher revient - tout comme l'exploitation touristique des richesses du vanua - à tirer le meilleur parti d'un patrimoine collectif créé par Dieu et de droits transmis par les ancêtres. Peu d'insulaires admettent que la pêche pratiquée par les locaux au sein du qoliqoli puisse avoir un impact écologique négatif: la pêche légitimement pratiquée serait aussi, le plus souvent, respectueuse du milieu marin, et la raréfaction de certains taxons (surtout signalée à Waya. Voir infra) est largement attribuée au comportement de pêcheurs venus de l'extérieur: non liés ou faiblement liés au vanua et aux mataqali locaux, ils agiraient pour cette raison sans respect ni mesure (Figure 6).

Ainsi, la pêche commerciale ne représente pas qu'un pis-aller face au développement du tourisme et au travail salarié : elle est considérée comme un complément lucratif ou même une alternative désirable, et comme l'un des seuls véritables moyens d'élévation économique et sociale. D'après un pêcheur de Vuake pratiquant activement la chasse sous-marine : "If you're a good diver, it's one of the easiest money you can get " (M., 36 ans). S'il n'est pas reconnu possible de gagner de fortes sommes d'argent avec les produits de la terre, la mer en revanche offrirait constamment, à qui sait les prendre, des richesses du vanua convertissables en dollars et en biens de consommation importés. 
Figure 6 : Le vanua rassemble la terre, la mer et les hommes en un tout indissociable

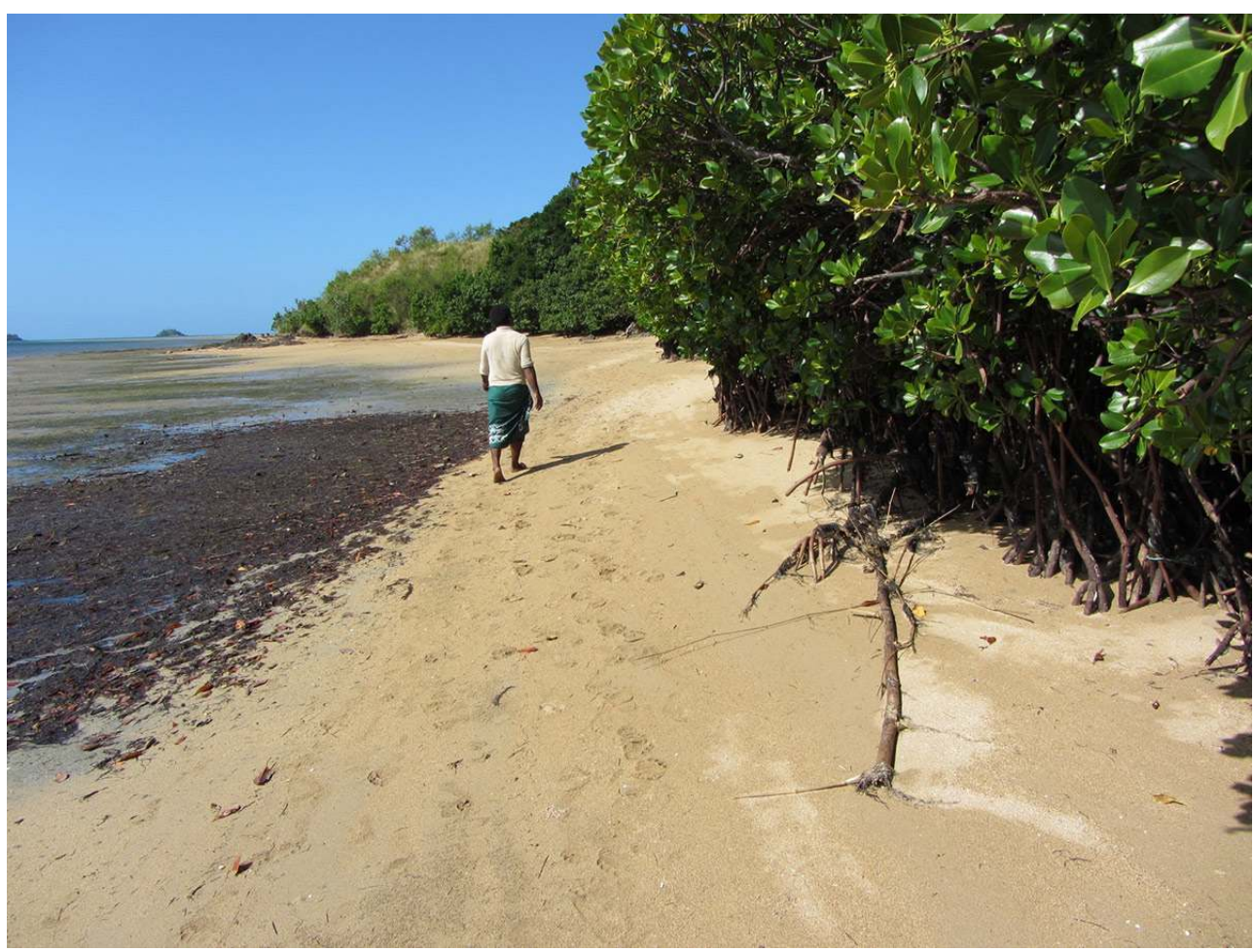

Bord de mer et mangrove à Vuake

(c) É. Nolet 2012

\section{Une société se transforme.... ou pas}

Si la pêche commerciale est loin de faire la fortune du plus grand nombre, chaque village a vu émerger ces dernières années des figures d'entrepreneurs enviés et influents. Leurs moyens financiers supérieurs à la moyenne leur permettent de contribuer avec une certaine largesse lors des festivités ou pour des projets locaux (réfection d'église, etc.), ce qui leur vaut alors la considération de leurs pairs. Mais ces hommes ont surtout la capacité de permettre aux autres villageois d'accéder à des revenus monétaires en les associant à leurs expéditions ou en leur louant leurs bateaux de pêche, acquis à grands frais ${ }^{17}$. Il est admis que réussir dans le domaine de la pêche implique un équipement de type occidental performant et l'accès à un bateau doté d'un moteur puissant, ardemment désiré par toutes les familles :

«Here, in our village, if I'm going fishing, they see me going fishing, they want to go too. [...] Because I've got the stuff. I've got the engine for the boat. They come. Like that. But to their own, they can't » (N., 50 ans, Yalobi).

Des habitants ont même la possibilité de devenir des intermédiaires (middle-men), revendant à Viti Levu ou aux grands resorts des Mamanuca du poisson acheté pour un prix inférieur aux pêcheurs de leur île.

Si la pêche marchande est loin de bouleverser les équilibres socio-hiérarchiques établis par la parenté, l'histoire des migrations et la chefferie, elle contribue à enraciner dans la vie locale des formes non traditionnelles d'échange (entre villageois et middle-men, pêcheurs et non pêcheurs, etc.) et renforce la position d'individus capables de réaliser 
une synthèse efficace entre des compétences ou vertus vues comme "autochtones " (savoirs coutumiers relatifs à la mer, générosité cérémonielle) et «importées » (esprit d'entreprise, aptitude à thésauriser, anticiper). Les commentaires qu'ils suscitent sont à la mesure de leur réussite : des villageois pensent que les patrons de pêche et middle-men profitent de manière abusive d'une ressource collective, banalisent des systèmes d'aspirations propres aux kai valagi (occidentaux blancs) au village ou jouissent d'un ascendant déplacé dans la communauté (Veitayaki 1995: 67-68), quand d'autres les admirent et aspirent à leur ressembler.

Le fait que l'entreprenariat et l'enrichissement personnel soient plus aisés que dans le passé n'empêche pas la persistance de puissants mécanismes de coopération, la pêche commerciale et le tourisme permettant aussi de pourvoir à des besoins collectifs pour le village et les clans. Le conseil de village de Vuake a ainsi interdit la pêche sur une partie $\mathrm{du}$ qoliqoli pour un an dans le but de favoriser la régénération d'espèces commercialisables et de susciter des rentrées d'argent pour les frais scolaires. Plus généralement, on observe que les principes de l'économie de marché coexistent avec des règles de redistribution communautaire qui forcent, dans une certaine mesure, à un partage des profits. Tout d'abord, les expéditions de pêche ne mobilisent que rarement un seul individu et font le plus souvent intervenir des frères, amis ou cousins qui profitent $\mathrm{du}$ fruit de la vente (Figure 7). Agir en commun et partager serait une force et une garantie de plus haute efficacité : « we go with the gang, like five [...] we get more. We sell the fish, and we share the money ». Une femme de Vuake expliquait en outre que les levées de fonds festives connues à Fidji sous le nom de soli étaient un moyen de faire circuler les profits, d'aplanir les différences générées par la vie économique et de faire montre de pouvoir et de réussite individuelle :

"If I'm making mud crabs and have money. They know. She has money. [...] [They will say] 'You have to go there! Come! Come! Come you go there. You go and soli'. 'Yeah, I come'. My mind: we collected this. So, OK. [...] I can give much more than what they give. To show that I have plenty money » (R., 50 ans, Vuake).

Un habitant de Vuake affirmait pour sa part être «toujours chanceux » à la pêche en raison du soin mis à redistribuer ses surplus aux proches parents, voisins, alliés (M., 36 ans). Inversement, « manger seul » alors que la pêche a été abondante expose un pêcheur à la honte (madua) et aux rumeurs. Ainsi la transformation socio-économique est loin d'être radicale: d'une part parce que les coutumes de partage se maintiennent et d'autre part parce qu'une partie des bénéfices dégagés grâce à la pêche commerciale est réinjectée dans des circuits d'échange et de compensations, qui contribuent à les rendre socialement acceptables (Veitayaki $1995: 13)^{18}$. Les ressources du vanua transformées en liquidités retournent toujours un peu au vanua, converties en relations sociales ou en biens collectifs. Il faut enfin remarquer que les situations d'autorité et d'influence acquises grâce à la pêche se construisent, mais aussi se déconstruisent: si plusieurs middle-men ont pu coexister à Yalobi, il n'y en avait plus qu'un seul au moment de l'enquête, les autres ayant accusé des pertes et étant « redevenus (simples) pêcheurs ». 
Figure 7 : Agir en commun, partager sa bonne fortune

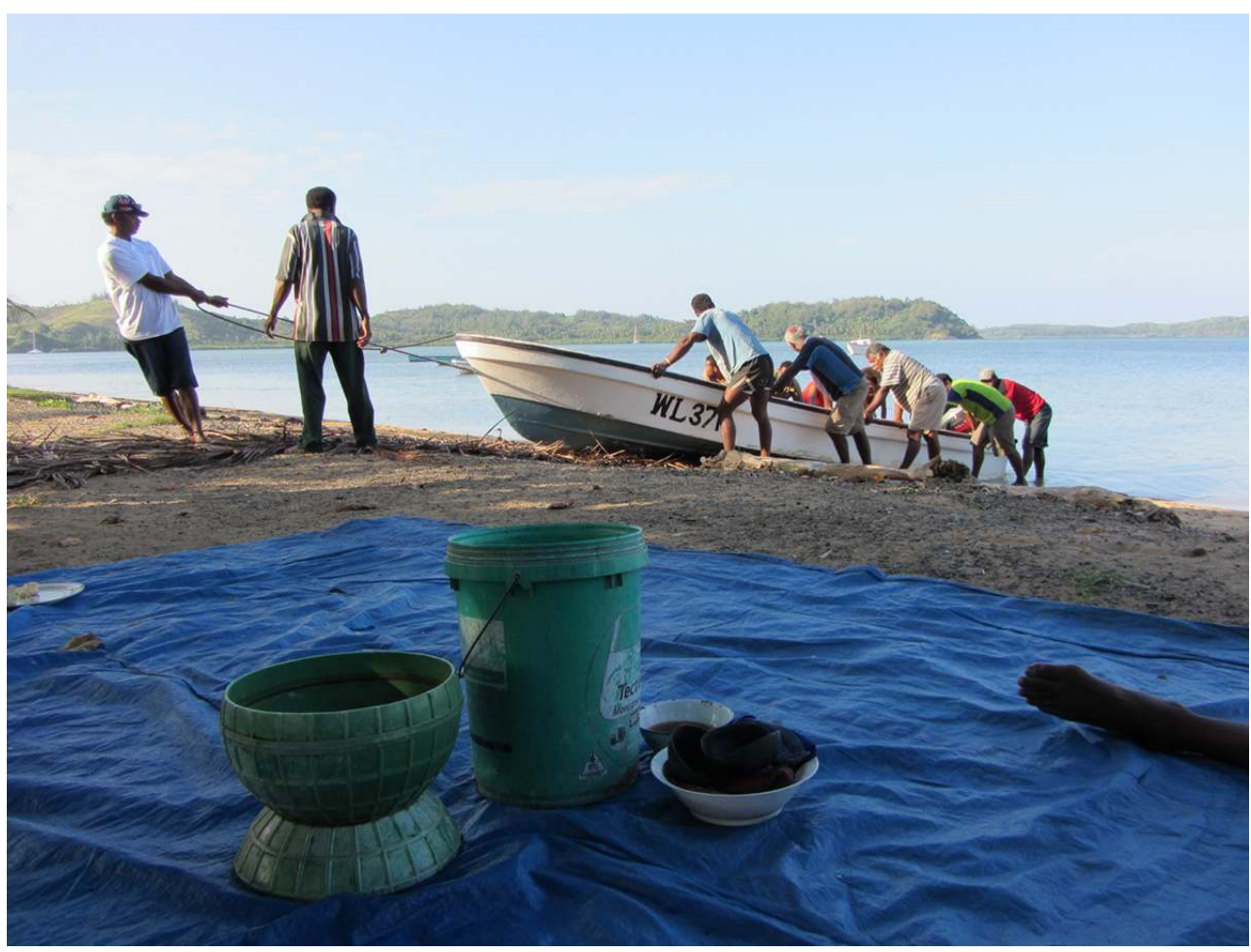

Pêcheurs hâlant un bateau à Vuake. Au premier plan, un plat à KAVA

(c) É. Nolet 2012 
Figure 8 : Le travail des jardins, un complément indispensable

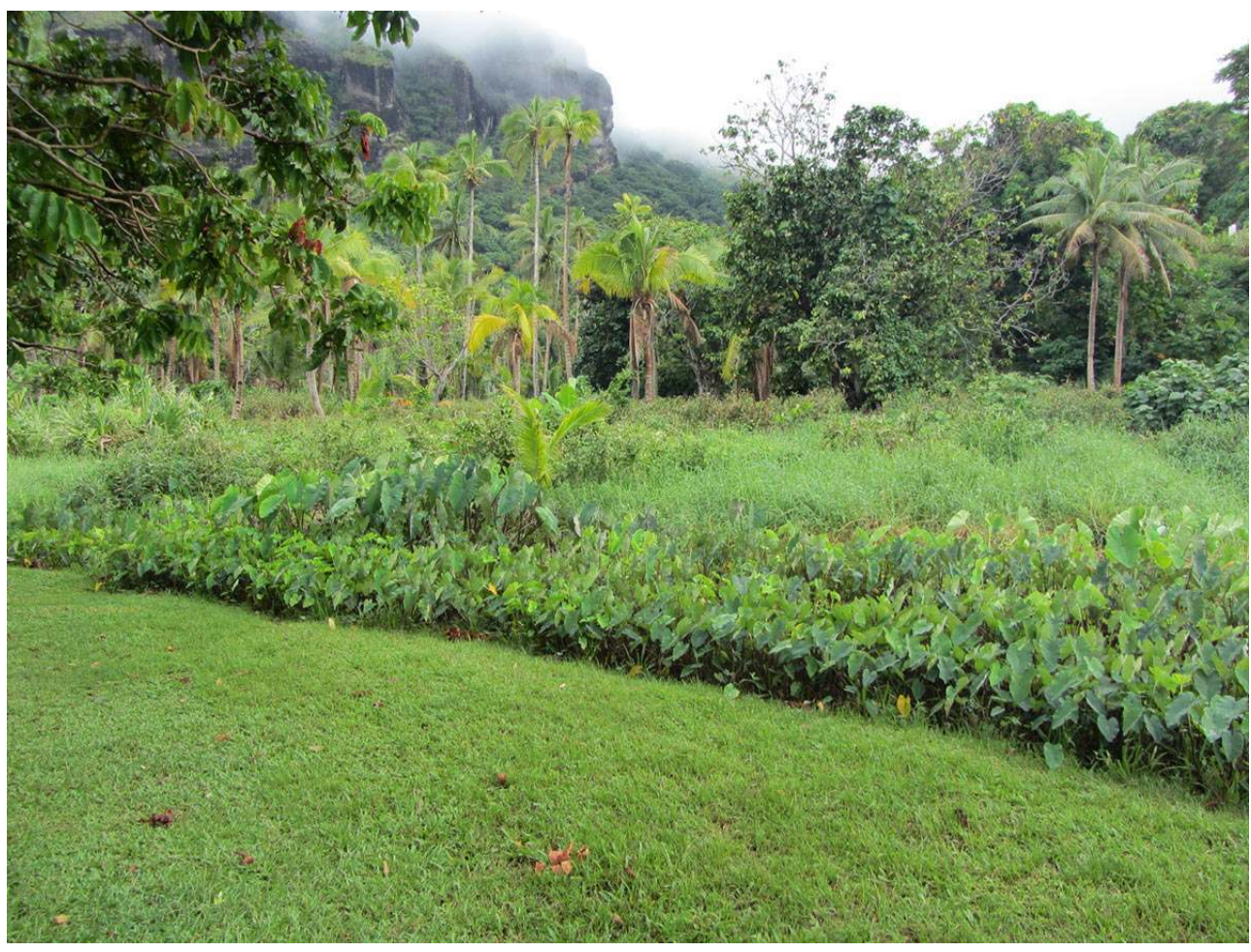

Plantation de taro à Yalobi

(c) É. Nolet 2012

30 On observera que même si les villageois tendent à avoir des activités de plus en plus spécialisées, beaucoup évoluent au gré des opportunités en faisant alterner des périodes longues ou brèves d'emploi au sein des resorts avec des périodes de vie au village centrées sur la pêche, les plantations, ou encore l'artisanat pour les femmes (Figure 8). Certains ont expliqué trouver un équilibre personnel dans cette polyactivité, les moments de vie au village, synonymes de forte participation sociale et culturelle, compensant l'éloignement véritable ou symbolique associé au salariat. De la même manière, aucun pêcheur ne délaisse entièrement les plantations, le travail agricole étant considéré comme un devoir masculin et un complément important des revenus monétaires générés par la pêche («it's a fifty-fifty policy» pour I., 50 ans, Vuake). Choisir entre terre et mer, entre tourisme et pêche, entre libéralisme et réciprocité communautaire ne serait ainsi pas une nécessité morale ou économique, la norme étant au contraire de faire cohabiter différents types d'activités, d'identités et d'engagements.

31 Nous allons maintenant envisager les perceptions et les pratiques de la conservation environnementale dans ces sociétés où la mer garde un rôle économique essentiel, autant pour la pêche que pour l'attractivité touristique. Voit-on s'exprimer des inquiétudes faisant écho aux débats actuels sur l'impact du tourisme, du changement climatique ou de la surpêche dans l'archipel fidjien? Les résidents ont-ils le sentiment d'une moindre biodiversité, de menaces écologiques ou de comportements abusifs, et quelles conséquences en tirent-ils au quotidien? 


\section{Préserver l'environnement marin : pourquoi, de quoi, comment?}

\section{Les représentations de l'état du milieu marin}

Les villageois sont nombreux à signaler des changements relatifs à la taille, aux quantités ou à la diversité des espèces pêchées, perçus à l'échelle de leur propre vie ou par référence aux souvenirs de leurs parents. Un résident de Yalobi a expliqué que les pêcheurs devaient à présent multiplier les pêches de nuit et pousser toujours plus loin leurs expéditions en mer pour obtenir une quantité de poissons suffisante (I., 40 ans). D'autres ont déploré l'apparition plus rare de taxons, comme la saumonée léopard ( Plectropomus leopardus) ou l'empereur mahsena, cabutu (Lethrinus mahsena). Des différences ont toutefois été relevées entre les témoignages recueillis à Yalobi et à Vuake. Les habitants de Waya, plus exposés aux conséquences des activités économiques de Viti Levu (exploitation de la canne à sucre, industrie, activité portuaire, etc.), sources de pollution et de circulation maritime effrénée, dénoncent des perturbations plus fréquemment et avec davantage de force. Ceux de Vuake se sont montrés en général plus sereins et confiants, en évoquant la stabilité ou la haute capacité de résilience de leur milieu marin. Si I., 50 ans, expliquait qu'il était désormais plus difficile de pêcher au fusil en raison d'une diminution de la taille des poissons, d'autres résidents ont affirmé «ne pas voir de différences ». Plusieurs ont même estimé que l'impression de moindre biodiversité marine était une illusion engendrée par le tourisme : les insulaires seraient devenus incapables de comprendre, nommer et prélever de manière efficace les créatures du vanua, concluant improprement à une déplétion des ressources.

Cette croyance répandue à Vuake existait aussi à Waya, un pêcheur prétendant même qu'il y avait « plus de poissons » que dans le passé mais que les insulaires étaient devenus trop « paresseux » ou ignorants pour en tirer avantage (N., 50 ans, Yalobi). Un pêcheur aguerri de Vuake affirmait pour sa part que les villageois ne savaient plus repérer ni anticiper les mouvements des poissons : des changements saisonniers d'habitat, ou des déplacements consécutifs aux tempêtes ou à la multiplication des bateaux motorisés seraient interprétés à tord comme une raréfaction ou disparition :

« Sometimes when it's low tide, then you go and put the net here [...], no! The fish they are already gone. You have to wait for the tide to come in, then the fish comes back, than you can see the fish » (E., 54 ans).

Pour certains l'environnement marin de Vuake serait ainsi par nature riche et productif mais ne révèlerait cette richesse qu'aux sachants de la tradition. Une pêcheuse considérait même que la situation géographique de Matacawalevu au sein des Yasawa contribuait à attirer de grandes quantités de poissons vers cette île: les bancs s'y dirigeraient naturellement puis seraient retenus aux abords, comme à l'intérieur d'une nasse (fish trap).

En résumé, le sentiment de vivre une mutation écologique est diversement ressenti à Vuake et Yalobi. Cette variabilité est susceptible de refléter plusieurs catégories de paramètres, comme l'expérience inégale des pêcheurs, l'utilisation d'un matériel et de techniques différents ou l'exploitation de zones de pêche plus ou moins vastes et riches (voir à ce sujet les observations de Turner et al. (2007 : 1005) dans les îles Lau, Fidji Est.). On notera cependant que le sentiment d'une bonne conservation générale de 
l'environnement est, à Vuake, partagé par des pêcheurs aux profils très divers : hommes et femmes, investissant la haute mer ou cantonnés aux abords de l'île, revenus récemment ou ayant plusieurs décennies de pratique locale à leur actif. On peut raisonnablement penser, comme le discours des habitants de Yalobi y invite, que la proximité de Viti Levu est en partie responsable des dégradations observées à Waya, Matacawalevu étant mieux préservée de part sa position plus septentrionale. D'un autre côté, il semble que l'opinion des habitants de Vuake s'appuie aussi sur des représentations de l'environnement local, vu comme naturellement prodigue (contrairement à celui de la proche île de Nacula, jugé « sec » et « vide » à Vuake) et sur le sentiment d'un assez faible impact écologique des comportements de subsistance locaux ou des resorts voisins.

\section{La hiérarchie des causes}

Si les perceptions de l'état du récif divergent, une majorité d'habitants s'accorde sur l'idée que les altérations constatées sont surtout dues à des acteurs et à des phénomènes exogènes, en particulier à la pratique d'une pêche commerciale agressive par des entreprises étrangères ou des populations de Viti Levu mal dotées en terres. L'influence de la surpêche, liée à la délivrance d'un trop grand nombre de permis ${ }^{19}$, au non-respect des limites légales des qoliqoli, à l'expansion démographique, au braconnage, voire au fait que les femmes "pêcheraient davantage que dans le passé » a été mise en cause : «So many people are using our sea! » se lamentait ainsi I., 40 ans, à Yalobi. Par exemple, la collecte intensive des holothuries aurait, de l'avis d'habitants de Waya, été un élément de perturbation des chaînes trophiques, agissant sur tout l'écosystème du récif. D'autres ont évoqué l'impact du passage de multiples cargos et bateaux de plaisance dont les rejets de carburant et d'huile à moteur endommageraient le corail, ou encore l'envahissement des plages par des déchets venus des villes côtières de Viti Levu.

L'impact d'activités locales comme le défrichement de la mangrove ou l'élevage de porcs en bordure de mer a été discuté, mais leur rôle n'a jamais été envisagé comme central. De même, la responsabilité du changement climatique - dont une large partie des insulaires a entendu parler via la radio, la télévision et des réunions d'information d'ONG - n'était pas mentionnée ou reléguée au second plan dans la hiérarchie des causes. L'avidité d'acteurs extérieurs, aggravée par le manque de surveillance ou le laxisme de l'administration territoriale, mais aussi la course au profit pratiquée sur la grande île expliqueraient, en premier lieu, les maux ressentis aux Yasawa :

« For us, we do our part. [...] But the main thing is all these fishermen coming from the mainland, because they come in the day time, they come in the night time. [...] Some they've got licence, some they don't have any licence » (T., 50 ans, Yalobi).

Le tourisme en revanche n'a jamais été présenté comme une source majeure de dégradations. Si des habitants ont signalé des nuisances liées au passage des bateaux de croisière ou ont déploré la création de passes artificielles et de remblais à l'usage des resorts, le tourisme est aussi vu comme une force positive en matière de protection de la nature. Le besoin de préserver la beauté naturelle des îles et des espaces marins devenue presque aussi cruciale que la fécondité des terres, car ce sont les lagons miroitants, les plages blanches, les fonds poissonneux qui attirent les touristes inciterait à contrôler plus étroitement les dépôts d'ordures, tandis que certains resorts de luxe passent pour être de véritables modèles en termes de tourisme durable. Beaucoup d'insulaires considèrent en outre que les dommages environnementaux ou sociaux 
engendrés par le tourisme sont un moindre mal au regard de son influence positive sur l'économie locale.

\section{Surveiller et sanctionner}

39 Même si les représentations de l'état de l'environnement marin diffèrent, une large majorité d'habitants s'accorde sur l'importance de préserver ou d'instaurer des mesures conservatoires, en particulier pour limiter la surpêche et la pêche illégale au sein du qoliqoli. La solution serait d'abord politique et nationale et impliquerait un meilleur contrôle du qoliqoli par le pouvoir d'État ou avec des moyens de sanction délégués par l'État. Certains prônent un durcissement des amendes pour les pratiques de pêche illégales, ou voudraient que des villageois sélectionnés puissent exercer en tant que gardes-pêche (fish wardens), autorisés à contrôler les pêcheurs de passage et à saisir leurs prises en cas d'infraction. Cette appropriation par les villageois d'une petite portion du pouvoir de répression étatique est généralement perçue comme efficace, même si contrôler les vastes qoliqoli des Yasawa s'avère être une véritable gageure.

40 Tous les habitants affirment aussi prendre des précautions à leur propre niveau, sans être forcément toujours très convaincus de leur utilité en termes de protection de la nature et en mettant au premier rang la crainte d'amendes infligées par le gouvernement. Les pêcheurs de Vuake et Yalobi ont expliqué s'interdire par exemple l'usage des bouteilles d'oxygène, du poison issu de l'écorce du tuva (Derris trifoliata) ou de filets à mailles fines (moins de 3 pouces, soit environ 7,6 cm) - même si, d'après des agents du Département des Pêches rencontrés à Yalobi certaines de ces méthodes, sous la pression sans cesse croissante du marché, seraient en réalité employées. D'autres ont dit se plier à la règle du rejet des jeunes poissons dans un but de conservation et détruire systématiquement l'acanthaster pourpre, bula (Acanthaster planci), une étoile de mer corallivore dangereuse pour le récif.

41 Les villageois admettent pourtant que certaines règles ou recommandations sont moins faciles à respecter que d'autres. Les tortues, ikabula (Chelonidae), dont la viande est très goûtée, sont toujours chassées en dépit des restrictions, et leurs œufs seraient aussi occasionnellement consommés. Il serait tout aussi malaisé d'obtenir que les pêcheurs cessent de profiter des grands rassemblements de kawakawa (un terme qui regroupe plusieurs espèces de Serranidae) qui se produisent durant la saison du frai et sont traditionnellement connus et exploités par les habitants de Fidji.

\section{Les aires marines gérées localement : une solution?}

42 La solution consistant à interdire ou limiter temporairement la pêche dans une partie du domaine marin soulève quant à elle des réactions contrastées. À Fidji et dans d'autres pays d'Océanie, de nombreuses communautés - souvent sous l'impulsion de leurs gouvernements ou d'institutions promouvant une gestion durable des ressources - ont été amenées à réactiver, étendre ou adapter des procédures d'origine pré-européenne de gestion des ressources marines, fondées en particulier sur l'utilisation des restrictions (tabous) de pêche (Ruddle \& Johannes 1989, Johannes 2002, Bambridge 2016). Ces démarches peuvent être motivées au départ par le constat d'une diminution des stocks ou de la raréfaction d'espèces, menaçant l'économie locale (Aalbersberg et al. $2005: 144$ ). La réactivation, le renforcement ou la réinterprétation de procédés traditionnels de gestion 
des ressources sont aujourd'hui considérés comme un élément de solution au service de la conservation marine, mais aussi des besoins de développement des populations insulaires et côtières d'Océanie (Cornier \& Leblic 2016: 119) ${ }^{20}$. On observera que les projets de conservation attentifs aux pratiques et savoirs coutumiers peuvent s'appuyer sur l'idée que ceux-ci seraient spontanément écologistes et sur une approche "simplifiée » des réalités culturelles locales, occultant des phénomènes cadrant mal avec les objectifs de protection de la nature (Keller 2009: 83, Smith \& Wishnie 2000: 493), minimisant la flexibilité et l'historicité des modes de tenure foncière et maritime ou réduisant aux nécessités de conservation des procédures comme le tabou, en fait complexes et multiformes (Foale \& Macintyre 2000, Foale et al. 2011, Artaud 2014).

À Fidji, le principal acteur de la promotion de la gestion communautaire des ressources marines est le Réseau Fidjien des Aires Marines Gérées Localement (Fijian Locally Managed Marine Area Network ou FLMMA Network), fondé en 2001. Ce réseau, qui associe un ensemble de partenaires locaux et internationaux (scientifiques, communautés locales, organisations gouvernementales et non-gouvernementales), encourage les communautés à mettre en place des "aires marines gérées localement» (AMGL ou en anglais locally managed marine area) ${ }^{21}$. Au sein des AMGL, les populations développent des pratiques conservatoires qui peuvent prendre la forme de tabous (en fidjien tabu) temporaires ou déplacés de zone en zone, de restrictions sur l'usage de certains engins ou sur la pêche de certaines espèces, d'interdits de pêche saisonniers ou plus pérennes (Govan et al. 2008 : 2-3, Hastings et al. 2015). Le Réseau Fidjien des Aires Marines Gérées Localement s'oriente vers des objectifs de conservation biologique tout en promouvant une plus forte autonomie des communautés dans le processus de gestion et de conservation des ressources, ainsi que les savoirs écologiques locaux ${ }^{22}$. Le Département des Pêches du gouvernement fidjien et ses partenaires s'offrent en conseillers et facilitateurs, mais ne sont pas censés décider à la place des acteurs locaux de l'opportunité de créer ou rouvrir une AMGL.

Tout en ancrant sa légitimité dans une "tradition" (inégalement documentée par l'ethnographie) qu'il a aussi vocation à valoriser et protéger (Govan et al. 2008:8), le concept d'AMGL implique des innovations, voire construit un nouveau mode d'interaction avec l'environnement local. Les projets collaboratifs de création et de gestion des AMGL peuvent d'abord combiner les restrictions avec des méthodes scientifiques modernes, notamment pour l'évaluation des stocks de poissons (Aalbersberg et al. $2005: 146$ ), qui introduisent de nouvelles manières d'appréhender les espèces et les rythmes marins. Ces projets peuvent en outre perturber ou renouveler les chaînes de décision à l'échelle communautaire. B. Aalbersberg et al. évoquent la demande, formulée par un groupe d'experts de l'University of the South Pacific de Fidji travaillant à la création d'une aire protégée à Ucunivanua (Verata), d'une représentation à part égale des hommes, femmes et jeunes au sein du groupe chargé de gérer la zone tabu (ibid: 145). Dans une société où la plus grande partie du pouvoir décisionnel incombe aux hommes et aux chefs, une telle approche, fondée sur l'idée qu'une bonne gestion de l'environnement repose forcément sur la participation de tous et sur la collégialité, est en rupture avec les habitudes locales. Dans le cas de l'aire tabu Lamiti-Malawai sur l'île de Gau, É. Fache a pourtant pu remarquer une faible efficacité des initiatives visant, depuis une dizaine d'années, à accroitre la participation des femmes (Fache \& Breckwoldt 2018: 258-259). La mise en place d'une AMGL implique donc une communauté et sa (ou plutôt « la ») tradition, mais aussi l'irruption de nouvelles manières de voir et de faire, tendant vers la création 
d'institutions hybrides ou encore modifiant le mode et le champ d'application de procédures déjà existantes (ibid: 262).

À Vuake et Yalobi, les expériences antérieures de clôture d'une portion du qoliqoli sont largement décrites comme des succès. Ces tabous temporaires prononcés sur les conseils de représentants de l'État (« on a voulu essayé », «let's try, let see mada!»), se seraient révélés efficaces, en permettant de profiter d'une pêche fabuleuse lors de la réouverture ( tara) et, d'après quelques témoins, en ramenant des "gros poissons" aux abords du village (Thaman et al. 2017). Néanmoins, tous ne s'accordent pas sur la position géographique, la nature et la durée des tabous de pêche à prononcer désormais, ni sur le caractère impérieux de telles restrictions.

Les habitants qui ont reçu une éducation citadine ou qui ont été employés dans l'administration territoriale sont en général sensibilisés aux questions écologiques et favorables aux interdits de pêche. Pour d'autres en revanche, convaincus de la bonne santé ou du pouvoir de résilience du récif, une telle démarche serait inutile : «Why we had to make the taboo? There are millions and millions of fish here » estimait A., 45 ans, à Vuake. De telles réserves existaient aussi à Yalobi, en dépit des dommages constatés dans l'environnement local. Une partie des habitants a estimé que les restrictions n'étaient intéressantes qu'à court terme et à une échelle restreinte, avant tout parce qu'elles étaient susceptibles de générer des inégalités sociales. La constitution de zones tabu aux abords des villages obligerait en effet les pêcheurs à pousser toujours plus loin leur exploration de la mer et offrirait (une fois de plus) un net avantage aux possesseurs de bateaux, tout en lésant les autres résidents. Ceux qui ont des difficultés à se déplacer et à rechercher d'autres voies d'accès à la mer, comme les personnes âgées, les infirmes, seraient particulièrement désavantagés.

À Fidji, la distribution des droits fonciers reflète l'histoire des migrations, des guerres et des négociations politiques intervenues avant et sous la période coloniale, ainsi que les rôles cérémoniels des unités sociales (Overton \& Banks 1988 : 126, Sahlins 2004). L'accès aux ressources du qoliqoli, appropriées à l'échelle d'une yavusa ou d'un vanua enregistré(e) en tant qu'« unité propriétaire " par le gouvernement fidjien, contribue (avec d'autres mécanismes, comme l'emploi urbain) à compenser, un peu, l'iniquité foncière : chaque pêcheur ou pêcheuse peut en effet espérer grâce à l'intensité de son labeur, à la précision de ses connaissances, à la qualité de ses alliances, faire vivre sa famille ou améliorer son sort - surtout depuis que le tourisme facilite la mise en marché des ressources marines (voir Veitayaki et al. 2007 : 302). Pour des villageois, il est ainsi vital que les mesures conservatoires n'empêchent pas sur le long terme un accès équitable au domaine marin et que ce dernier puisse continuer à limiter les écarts de richesses, ou encore à prodiguer les mets d'origine marine requis pour les accueils ou d'autres cérémonies.

Certains suggèrent que l'activation ou l'extension de pratiques coutumières de gestion des ressources ne seraient pas adaptées aux conditions actuelles, marquées par une élévation du coût de la vie et par une injonction à disposer de toujours plus de ressources monétaires, sous l'effet de la mondialisation, de l'économie de marché, et même du tourisme. D'autres redoutent visiblement qu'un dispositif comme l'AMGL, vu comme « exogène » et non pas local, puisse conduire à l'institution d'interdits inamovibles, à l'appropriation du domaine marin par des intérêts extérieurs, ou à une forme plus globale d'ingérence étatique dans les affaires communautaires. Des villageois, enfin, ont évoqué l'impossibilité d'exercer une surveillance continue sur les zones protégées et de les 
préserver de la convoitise des fraudeurs (en particulier de pêcheurs venus de l'extérieur), poussés par la misère ou par l'appât du gain : là encore, il serait inévitable de voir certains " souffrir » et d'autres « profiter » (voir Hastings et al. 2015 : 155-156).

En 2012, les habitants de Vuake ne considéraient pas la mise en place d'un tabou permanent sur une portion du récif (marine reserve, marine protected area), afin d'améliorer les conditions de la pêche par un effet de "vases communicants " (spillover effect) comme nécessaire, tandis que ceux de Yalobi jugeaient l'idée peu réaliste au vu de la petite taille de leur qoliqoli et de conditions déjà très concurrentielles. Certains étaient toutefois prêts à accepter un tabou semi-permanent visant à créer une réserve locale pouvant être ouverte pour répondre à des besoins collectifs : par exemple nourrir un groupe d'agents du gouvernement en visite sur l'île ou organiser des festivités religieuses - un intérêt commun clairement identifié étant vu comme un préalable. Tous ne s'accordaient pourtant pas sur les limites de la zone à protéger ou sur les modes de gestion à mettre en place.

D'après T., du Département des Pêches - rencontrée en juin 2012, lors d'une mission de sensibilisation aux risques écologiques à Yalobi - l'un des principaux obstacles à surmonter pour créer une aire protégée serait en définitive l'absence de consensus, particulièrement lorsqu'il existe des conflits sur la succession aux titres de chefs et des factions politiques rivales. "If there are differences in the village, it's hard to coach». La vacance du pouvoir coutumier ou la contestation d'une autorité en place peuvent conduire, selon les cas, à une situation de statu quo (avec des partis rivaux, revendiquant chacun la légitimité à prononcer les tabous) ou encore, dans le cas d'aires protégées déjà existantes, au non-respect des interdits de pêche (Müehlig-Hofmann 2008).

\section{Respecter la coutume, obéir à Dieu}

51 On notera que de nombreux insulaires tendent aussi à penser que les possibilités d'action humaine sur l'état de l'environnement sont limitées. Les terres et les espaces marins ont en effet une nature donnée et désirée par Dieu, qu'il serait vain de chercher à transformer en profondeur, y compris au moyen des tabous :

"We don't have any taboo right here in Vuake. [...] God makes everything. We just come and use it and go. Our past generation used this fish and they died and we came again and our next generation will be coming again...There is no difference » (A., 45 ans, Vuake).

L'état de l'environnement serait avant tout le résultat d'une volonté divine, d'un grand partage ayant abouti à donner aux uns une abondance de ressources marines, à d'autres des terres fécondes, ou d'une beauté susceptible d'attirer en masse les visiteurs étrangers. Cet équilibre des origines ne saurait être aisément et même impunément perturbé.

Par ailleurs, des habitants ont expliqué que l'environnement marin a été conçu, offert par Dieu précisément pour que les hommes y trouvent une nourriture quotidienne. Exploiter, utiliser le milieu marin, même dans une perspective commerciale, serait aller dans le sens de ce que veut Dieu. Interdire totalement l'exploitation d'une portion du récif irait par contre contre le souhait divin d'un espace maritime ouvert, où chaque homme, femme et enfant peut librement puiser les moyens de sa subsistance. Il serait pour cette raison difficile de convaincre les communautés de pêcheurs de se tourner de façon prioritaire ou pérenne vers d'autres formes d'activités (artisanat, tourisme, couture, etc.) pour limiter les pressions exercées sur le domaine marin. D'après un intervenant de l'ONG Wildlife 
Conservation Society rencontré à Yalobi, le sentiment d'un droit traditionnel à profiter des ressources marines, ainsi que l'importance des gains générés par la pêche commerciale démobilisent les communautés :

«We should come in with alternative sources of livehood they can rely on [...] but it's very hard to communities that have lived all of their life fishing to change their livehoods, and to change their minset. It's a big challenge » (K., 30 ans, 2012).

Ce sentiment n'en exclut pas un autre, celui d'une responsabilité morale collective impliquant de préserver le patrimoine des "gens de la terre", fait de ressources naturelles mais aussi de noms, de titres, de récits oraux, d'objets typiques, de sites sacrés et d'autres traces mémorielles. Certains estiment que protéger le vanua repose d'abord sur une exploitation raisonnablement diversifiée des espaces terrestres et marins et dénoncent le danger d'une trop forte appétence pour les espèces les plus volumineuses, goûteuses et rentables, source de déséquilibres écologiques et sociaux. Pêcher ou collecter de manière opportuniste un ensemble de ressources, à la façon des ancêtres, et donc aussi redécouvrir ou transmettre les savoirs écologiques s'y rapportant, serait la première conduite à tenir. Inversement, refuser de consommer les blanches (matu, Gerres spp.) et autres menus poissons (Figure 9) ou encore les coquillages au goût fade des vasières, serait faire preuve d'arrogance et d'ingratitude, ne pas respecter le vanua.

Figure 9 : Un petit kabatia pêché par une enfant de Vuake

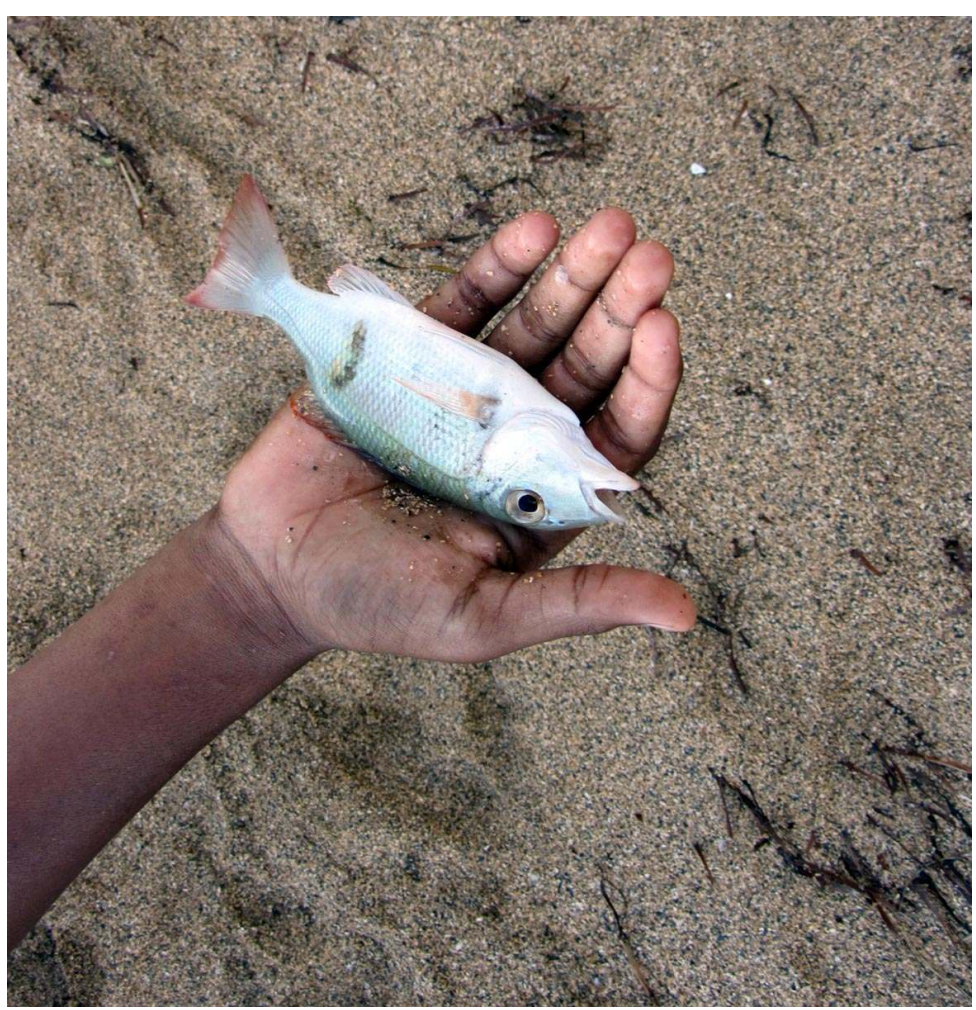

(c) É. Nolet 2012

Plus généralement, un ensemble de comportements individuels ou collectifs vont contribuer à préserver l'abondance naturelle, à contenter Dieu et à maintenir des liens harmonieux avec les entités spirituelles, qui continuent de jouer un rôle actif dans l'existence quotidienne des hommes (Ravuvu 1983 : 85-86, Tomlinson 2009 : 68). Partager les surplus de pêche, respecter les obligations cérémonielles et familiales, ne pas dilapider 
les ressources collectives ou drainer une trop vaste quantité de richesses locales à l'extérieur du vanua, ne pas faire souffrir inutilement les animaux pêchés, serait aussi nécessaire (et aussi traditionnel), voire davantage, que la stratégie du tabou qui est au cœur du dispositif de l'AMGL. Comme les cyclones et les inondations peuvent sanctionner les mauvaises conduites des hommes (Nolet 2016), la disponibilité des ressources marines est en partie le reflet du respect d'un ordre ancien liant les hommes entre eux et ces derniers aux esprits ancestraux, à Dieu et à ses créatures; un ordre dont les insulaires n'ont pas tous exactement la même vision, impliquant le respect des liens de parenté, de la chefferie, des valeurs chrétiennes.

La disparition ou la raréfaction d'un produit marin utile aux hommes pourra être considérée comme l'effet d'une prédation extérieure, d'une négligence de l'État dans sa mission de protection des communautés rurales, mais aussi comme une sanction ou un avertissement : par exemple si les membres d'une yavusa se déchirent sans fin dans des conflits de succession, si les chefs ne remplissent pas leurs devoirs de réciprocité à l'endroit du peuple (veiqaravi), si les rôles cérémoniels ne sont pas respectés et si les pratiques d'accumulation capitalistique prévalent outrageusement sur l'ethos du partage entre apparentés (Ravuvu 1983: 82, Brison 2007). En ce sens, parler de la mer et de l'appauvrissement des ressources (comme de la présence-absence des calamités naturelles) ouvre la voie à une forme de critique sociale et permet aussi bien de condamner l'évolution d'une partie de la société itaukei - soit les clans de la zone périurbaine de Nadi et de la capitale Suva, qui sont censés s'être " éloignés de leurs racines » et "vivre une vie dédiée à l'argent", bula vakailavo - que de pointer des disfonctionnements locaux.

\section{Conclusion}

57 Aux Yasawa, archipel longtemps resté en marge du développement économique fidjien, l'essor touristique est vu comme un juste retour de l'histoire : les insulaires présentent le tourisme comme une bénédiction («a blessing ») à une écrasante majorité, et se disent fiers d'incarner le " rêve océanien » ou la totalité nationale « fidjienne » pour les visiteurs étrangers. Les effets du tourisme ne sont pourtant pas homogènes dans les îles et villages de ce groupe insulaire où certains habitants, n'ayant pas accès aux emplois et aux revenus des resorts, continuent de dépendre fortement du milieu marin. Cet article a montré que si le tourisme contribue à un affaiblissement de la pêche de subsistance, il participe aussi paradoxalement au développement de la pêche commerciale: les insulaires qui ne peuvent ou ne veulent pas travailler dans les resorts peuvent, par la vente de produits marins soigneusement sélectionnés, tirer également profit de la présence des touristes, en tissant avec eux des liens d'échange directs et indirects. La mer continue ainsi d'être perçue comme une ressource collective, jouant son rôle nourricier via la mise en marché de produits marins, ou en tant que produit d'appel de l'industrie touristique.

Plus lucrative que l'emploi au sein des resorts, la pêche commerciale permet aussi à un individu d'échapper aux hiérarchies professionnelles et de conserver la possibilité d'une certaine polyactivité (pêche, agriculture, travail domestique, participation aux tâches collectives et aux cérémonies) et les moments de repos (vakacegu), d'attente, d'ennui, de sociabilité villageoise indispensables à l'équilibre d'une vie : le temps du travail est alors défini par les rythmes de la mer et les stratégies du pêcheur, et non par une organisation extérieure qui, dit-on, met l'employé sous pression, voire l'éloigne de sa vraie nature 
(Figure 10). Tout en étant perçu comme une chance, le tourisme est en effet source de contraintes et contribue à des changements socioculturels que la pêche commerciale peut à la fois accentuer (par exemple en favorisant le développement de relations marchandes entre villageois) ou contribuer à limiter et contrôler (en permettant à ceux qui bénéficient le moins du tourisme d'accéder aussi à des revenus monétaires, ou encore aux employés de resorts de revenir vivre au village).

Figure 10 : VAKACEgU (se reposer) : indispensable à l'équilibre d'une vie

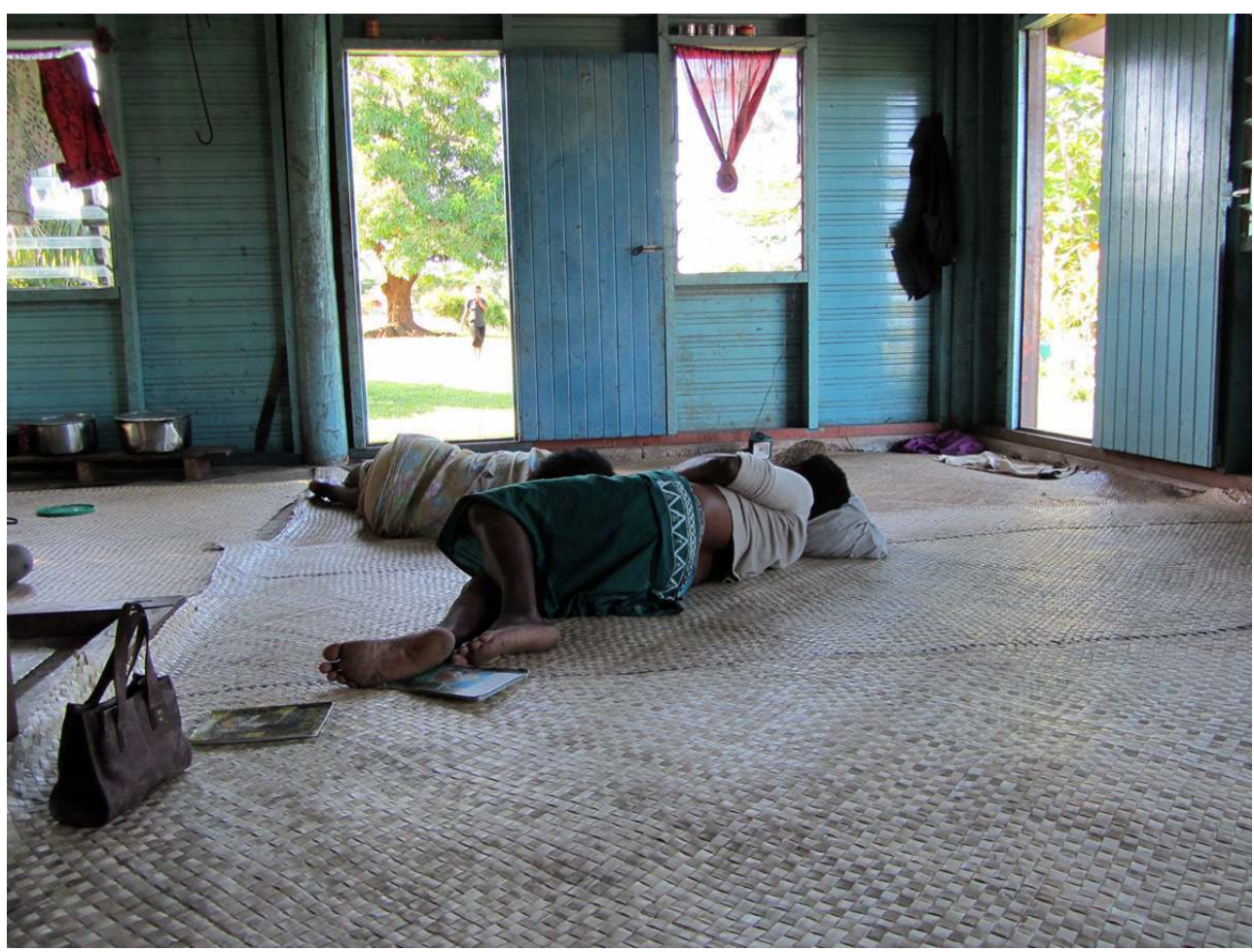

L'heure de la sieste dans une maison de Vuake

(c) É. Nolet 2012

Si le tourisme et la pêche commerciale tendent à modifier les rapports avec le milieu marin et les relations sociales au sens large, ces activités permettent aussi de renforcer des structures ou des relations existantes, ou bien sont régulées par des normes sociales inscrites dans la tradition locale. Ainsi, l'argent gagné grâce au tourisme aidera à accéder au confort moderne, mais aussi à s'insérer positivement dans la société locale au travers des prestations cérémonielles - achat de nattes, dents de cachalot ou tabua, coupons de coton et autres biens échangés lors de diverses cérémonies -, de contributions généreuses à des projets collectifs et d'autres paiements. Par ailleurs, si vendre les produits du qoliqoli n'est pas condamnable en soi, il importe que les pêcheurs sachent préserver une forme d'éthique villageoise et redistribuer les fruits de leur bonne fortune pour conserver l'estime des leurs et consolider leur position.

De la même manière, si la multiplication des appels à la vigilance, les projets de sensibilisation aux risques et l'observation concrète de changements écologiques contribuent à transformer les modes d'utilisation et de représentation de la mer, les pratiques de conservation doivent s'accorder avec les conceptions existantes du bienvivre et ne pas entraver la réalisation d'objectifs sociaux vus comme centraux, comme la 
préservation d'une certaine équité dans l'accès au domaine marin. De plus, on aperçoit que les pratiques conservatoires initiées par le gouvernement et le monde scientifique coexistent avec des représentations inscrites dans la tradition, qui impliquent une solidarité étroite entre le visible et l'invisible, les forces surnaturelles et les formes de la vie humaine, végétale et animale rassemblées dans l'entité indivisible du vanua. Préserver le milieu naturel peut certes passer par des " aires marines gérées localement » (AMGL), mais impose aussi de préserver des équilibres ou des dynamiques relationnelles essentiels, liant ces éléments.

61 Loin d'opposer pêche et tourisme, ou encore tradition et modernité, les habitants de Vuake et Yalobi envisagent en fait la situation d'avenir la plus positive au sein d'une combinaison de facteurs : le développement d'un tourisme de luxe, supposant l'affluence massive de capitaux étrangers et offrant un précieux marché aux pêcheurs locaux ; celui de resorts communautaires, dont les profits iraient entièrement aux membres du vanua et où les hiérarchies professionnelles seraient idéalement tempérées par le système d'obligations et de réciprocités socio-familiales; un renforcement par l'État des droits des communautés autochtones à exploiter, de manière prioritaire ou exclusive, les espèces marines présentes dans leur environnement immédiat ; enfin, des actions concrètes du gouvernement en faveur de la pêche commerciale (subventions à l'achat de bateaux en tout premier lieu), permettant à un plus grand nombre d'accéder aux espoirs et aux vertiges du «business de la mer ».

Je remercie chaleureusement les habitants de Yalobi et Vuake pour leur accueil et l'intérêt manifesté à mes recherches. Je remercie également le Labex CORAIL, Simonne Pauwels et le Centre de Recherche et de Documentation sur l'Océanie (CREDO, UMR 7308), ainsi que Joeli Veitayaki et Alifereti Koroilavesau qui m'ont grandement aidée à organiser les travaux de terrain. Merci enfin à Laurie Bouffandeau pour son aide au sujet des dénominations d'espèces marines.

\section{BIBLIOGRAPHIE}

Aalbersberg B., Tawake A. \& Parras T. 2005 - Village by village. Recovering Fiji's coastal fisheries. In : World Resources Institute, United Nations Development Programme, United Nations Environment Programme \& World Bank, World Resources 2005: The Wealth of the Poor - Managing Ecosystems to Fight Poverty. Washington, WRI : 144-151.

Abramson A. 2004 - A small matter of some rent to be paid. Toward an analysis of neo-traditional direct action in contemporary Fiji. In : Van Meijl T. \& Miedema J. (Ed.), Shifting Images of Identity in the Pacific. Leiden, KITLV Press : 75-99.

Adams W.M. \& Hulme D. 2001 - If community conservation is the answer in Africa, what is the question ? Oryx 35 (3) : 193-200.

Artaud H. 2014 - De l'« efficacité » symbolique des interdits à leur fonctionnalité écologique. Variations sur le « tabou » en milieux maritimes. Revue d'Ethnoécologie 6 [En ligne] URL : https:// journals.openedition.org/ethnoecologie/2055; consulté le 13 novembre 2018. 
Bambridge T. (Ed.) 2016 - The Rahui. Legal Pluralism in Polynesian Traditional Management of Ressources and Territories. Canberra, ANU Press, Pacific Series, $269 \mathrm{p}$.

Bose A. \& Fraenkel J. 2007 - Whatever happened to Western separatism? In : Fraenkel J. \& Firth S. (Ed.), From Election to Coup in Fiji: The 2006 Campaign and its Aftermath. Canberra, ANU Press : 225-242.

Brison K. 2007 - Our Wealth is Loving Each Other. Self and Society in Fiji. Lanham, MD, Lexington books, $151 \mathrm{p}$.

Brutti L. 2018 - L'industrie touristique à Fidji entre management autochtone et marketing global. In : Nolet É., Lindenmann P. \& Dousset L., Médiations politiques en Mélanésie contemporaine. Marseille, Pacific-Credo Publications : 285-316.

Cochrane E. 2004 - Explaining Cultural Diversity in Ancient Fiji: The Transmission of Ceramic Variability. Honolulu, University of Hawaii, thesis, $422 \mathrm{p}$.

Comley J., Tuveng L., Crabbe M. \& Raines P. 2005 - Yasawas Islands Coral Reef Conservation Project. Coral Cay Conservation Ltd, $68 \mathrm{p}$.

Connell J. 2015 - Competing islands ? The Manamuca and Yasawa islands, Fiji. In : Baldacchino G. (Ed.), Archipelago Tourism, Policies and Practices. Farnham - Burlington, Ashgate : 183-197.

Cornier S. \& Leblic I. 2016 - Kanak coastal communities and fisheries meeting new governance challenges and marine issues in New Caledonia. In Fache E. \& Pauwels S. (Ed.), Fisheries in the Pacific. The Challenges of Governance and Sustainability. Marseille, Pacific-Credo Publications : 119-174.

Cumming R.L., Aalbersberg W.G., Lovell E.R., Sykes H. \& Vuki V.C. 2002 - Coral Reefs of the Fiji Islands: Current Issues. Suva, USP, IAS Technical Report 2002/11, 15 p.

Deane W. 1921 - Fijian Society or the Sociology and Psychology of the Fijians. London, MacMillan \& Co, $255 \mathrm{p}$.

Fache É. \& Breckwoldt A. 2018 - Small-scale managed marine areas over time: Developments and challenges in a local Fijian reef fishery, Journal of Environmental Management 220 : 253-265.

Farrelly T. \& Talatoka Vudiniabola A. 2013 - Kerekere and indigenous social entrepreneurship, SITES: Journal of Social Anthropology and Cultural Studies 10 (2) : 1-29.

Foale S., Cohen P., Januchowski-Hartley S., Wenger A. \& Macintyre M. 2011 - Tenure and taboos: Origins and implications for fisheries in the Pacific. Fish and Fisheries 12 (4) : 357-369.

Foale S. \& Macintyre M. 2000 - Dynamic and flexible aspects of land and marine tenure at West Nggela: implications for marine resource management, Oceania 71 : 30-45.

Gardner R. \& Pawley A. 2006 - Annotated list of local plant names from Waya Island, Fiji, Records of the Auckland Museum $43: 97-108$.

Gibson D. 2012 - The cultural challenges faced by indigenous-owned small medium tourism enterprises (SMTEs) in Fiji. Case studies from the Yasawa islands, The Journal of Pacific Studies 32 (2) : 102-126.

Gillett R. \& Moy W. 2006 - Spearfishing in the Pacific Islands. Current Status and Management Issues. Nouméa, SPC, Rome, FAO, 76 p.

Govan H., Aalbersberg W., Tawake A. \& Parks J. E. 2008 - Locally-Managed Marine Areas: A guide to Supporting Community-Based Adaptive Management. The Locally-Managed Marine Area Network, $65 \mathrm{p}$. 
Hackel J.D. 1999 - Community conservation and the future of Africa's wildlife, Conservation Biology 13 (4) : 726-734.

Hastings J. G., Orbach M. K., Karrer L. B. \& Kaufman L. 2015 - MMAS in Fiji. Coastal Management 43 (2) : 155-171.

Jennings S. \& Polunin N.V.C. 1996 - Fishing strategies, fishery development and socioeconomics in traditionally managed Fijian fishing grounds, Fisheries Management and Ecology 3 (4) : 335-347.

Johannes R. E. 2002 - The renaissance of community-based marine resource management in Oceania, Annual Review of Ecological Systems $33: 317-340$.

Keller E. 2009 - The danger of misunderstanding 'culture', Madagascar Conservation and Development 4 (2) : 82-85.

McNamara K. E. \& Limalevu L. 2011 - Contextualising Livelihoods in a Vulnerability and Adaptation Assessment: Case study of Soso village, Fiji. Suva, USP, PACE-SD, 29 p. (Occasional Paper ; 2).

Muehlig-Hofmann A. 2008 - Chefferies et autorités coutumières : principaux facteurs intervenant dans la gestion et la conservation communautaires des ressources marines, Ressources Marines et Traditions, Bulletin de la CPS $21: 31-44$.

Naval Intelligence Division 1944 - Pacific Islands vol. III: Western Pacific, Londres, Geographical Handbook Series.

Nolet E. 2016 - 'Are you prepared?' Representations and management of floods in Lomanikoro, Rewa (Fiji), Disasters, 40 (4) : 720-739.

Nunn P. D., Aalbersberg W., Ravuvu A. D. \& Mimura N. 1994 - Assessment of Coastal Vulnerability and Resilience to Sea-Level Rise and Climate Change. Case Study: Yasawa Islands, Fiji. Phase II: Development of Methodology. Apia, SPREP, $118 \mathrm{p}$.

Overton J. \& Banks B. 1988 - Rural Fiji. Suva, University of the South Pacific, 230 p.

Pawley A. 2011 - What does it take to make an ethnographic dictionary ? On the treatment of fish and tree names in dictionaries of Oceanic Languages. In : Haig G.L.J., Nau N., Schnell S. \& Wegener C. (Ed.), Documenting Endangered Languages, Achievements and Perspectives. Berlin, de Gruyter Mouton : 263-287.

Pawley A. \& Sayaba T. 1971 - Fijian dialect divisions: Eastern and Western Fijian. Journal of the Polynesian Society 80 (4) : 405-436.

Pietrusewsky M., Hunt T. L. \& Ikehara-Quebral R. M. 1997 - A Lapita skeleton from Waya island, Fiji, Micronesica 30 (2) : 355-388.

Pigliasco G. C. 2007 - The Custodians of the Gift: Intangible Cultural Property and Commodification of the Fijian Firewalking Ceremony. Honolulu, University of Hawaii, Ph.D. Dissertation, 790 p.

Raven-Hart R. 1956 - A village in the Yasawas (Fiji). The Journal of the Polynesian Society 65 (2) : 95-154.

Ravuvu A. 1983 - The Fijian Way of Life. Suva, Institute of Pacific Studies of the University of the South Pacific, $130 \mathrm{p}$.

Roué M. \& Bahuchet S. 2014 (Ed.) - Conservation de la nature : quel rôle pour les sciences sociales? Revue d'Ethnoécologie 6 [En ligne] URL : https://journals.openedition.org/ethnoecologie/1874, consulté le 30 octobre 2018.

Ruddle K. \& Johannes R. E. 1989 (Ed.) - Traditional Marine Resource Management in the Pacific Basin: An Anthology. Jakarta, Unesco-ROSTSEA, 410 p. 
Sahlins M. 2004 - Apologies to Thucydides. Chicago, The University of Chicago Press, 334 p.

Sloan J. \& Chand K. 2015 - A Review of Near Shore Fisheries Law \& Governance in Fiji. Suva, Siwatibau \& Sloan, $30 \mathrm{p}$.

Smith E. A. \& Wishnie M. 2000 - Conservation and subsistence in small-scale societies, Annual Review of Anthropology 29 : 493-524.

Teh L.C.L., Teh L.S.L., Starkhouse B. \& Sumaila U.R. 2009 - An overview of socio-economic and ecological perspectives of Fiji's inshore reef fisheries, Marine Policy 33 : 807-817.

Thaman B., Thaman R.R., Balawa A. \& Veitayaki J. 2017 - The recovery of a tropical marine mollusk fishery: a transdiciplinary community-based approach in Navakavu, Fiji, Journal of Ethnobiology 37(3) : 494-513.

Tomlinson M. 2009 - Efficacy, truth, and silence: language ideologies in Fijian Christian conversions. Comparative Studies in Society and History 51 (1) : 64-90.

Toren C. 1999 - Drinking cash. The purification of money through ceremonial exchange in Fiji. In Toren C., Mind, Materiality and History, Explorations in Fijian Ethnography. London \& New York, Routledge : 27-44.

Turner R.A., Cakacaka A., Graham N.A.J., Polunin N.V.C., Pratchett M.S., Stead S.M. \& Wilson S.K. 2007 - Declining reliance on marine resources in remote South Pacific societies: ecological versus socio-economic drivers, Coral Reefs 26 (4) : 997-1008.

Twyman C. 2000 - Participatory conservation? Community-based natural resource management in Botswana. The Geographical Journal 166 (4) : 323-335.

Veitayaki J. 1995 - Fisheries Development in Fiji: the Quest for Sustainability. Suva, The University of the South Pacific, 233 p.

Veitayaki J., Tawake A., Bogiva A., Meo S., Ravula N., Vave R., Radikedike P. \& Fong P. S. 2007 - Addressing human factors in fisheries development and regulatory processes in Fiji: the Mositi Vanuaso experience, Ocean Yearbook 21 (1) : 289-306.

Weeks R. \& Jupiter S. D. 2013 - Adaptive comanagement of a marine protected area network in Fiji, Conservation Biology 27 (6) : 1234-1244.

Western D. \& Wright R. M. (Ed.) 1994 - Natural Connections. Perspectives in Community-based Conservation. Washington, Island Press, $600 \mathrm{p}$.

Williksen-Bakker S. 2002 - Fijian business - a bone of contention. Was it one of the factors leading to the political crisis of 2000? The Australian Journal of Anthropology 13 (1) : 72-87.

World Bank (The) 2017 - Republic of Fiji Systematic Country Diagnostic. Washington, International Bank for Reconstruction and Development - The World Bank, 74 p.

\section{NOTES}

1. En 2017, Fidji a officiellement accueilli 842884 visiteurs surtout venus d'Australie, de NouvelleZélande, d'Asie et des États-Unis, un chiffre record ([en ligne] URL : https://www.statsfiji.gov.fj/ index.php/statistics/tourism-and-migration-statistics/visitor-arrivals-statistics, consulté le 26 octobre 2018). Devenu la principale source de revenus du pays, le tourisme représenterait actuellement près de $38 \%$ du produit intérieur brut, d'après des chiffres diffusés par la Banque Mondiale (World Bank 2017 : xi). 
2. Des informations sur l'usage des ressources marines aux Yasawa ont été collectées à l'occasion d'études ethnologiques générales (Raven-Hart 1956) ou pour des recherches visant à évaluer les ressources de certaines îles et leur degré de vulnérabilité face au changement climatique (Nunn et al. 1994, McNamara \& Limalevu 2011). Sur l'exploitation de la mer dans d'autres régions et à Fidji en général, voir par exemple : Deane 1921, Veitayaki 1995, Jennings \& Polunin 1996, Turner et al. 2007, Thaman et al. 2017, Fache \& Breckwoldt 2018.

3. Voir Aalbersberg et al. 2005, Comley et al. 2005 et Weeks \& Jupiter 2013. Les politiques et enjeux de la gestion participative des ressources naturelles sont discutées par exemple dans Western \& Wright 1994, Twyman 2000 et Roué \& Bahuchet 2014.

4. Les deux îles sont situées respectivement au sud et au centre du groupe insulaire. Waya Levu bénéficie d'une plus grande proximité avec la ville de Lautoka à Viti Levu, mais ne possédait qu'un resort en fonctionnement et un autre en projet au moment de l'enquête en 2012. Matacawalevu n'accueillait aussi que deux structures de taille modeste, mais a l'avantage de se trouver dans une zone des Yasawa où le tourisme est particulièrement dynamique, avec des possibilités d'emplois dans plusieurs resorts à succès (Navutu Stars, Turtle Island, etc.).

5. Ces recherches ont été conduites dans le cadre du projet « Catastrophes et changement global à Fidji : impacts, représentations et pratiques sociales dans deux régions côtières ", financé par le Labex CORAIL. Les travaux de terrain ont reposé sur l'observation participante et la réalisation de 42 entretiens individuels dans les deux villages. L'auteure possède par ailleurs une expérience antérieure de 22 mois de terrain à Rewa (Viti Levu).

6. À Vuake et Yalobi, ce terme englobe en général les structures de type « backpacker " (destinées à des publics aux ressources limitées, typiquement de jeunes routards) dans le langage courant.

7. Le terme mataqali est généralement traduit par «clan» ou «sous-clan» en anglais. Les mataqali se composent de plusieurs tokatoka (famille étendue), plusieurs mataqali hiérarchisés formant une yavusa, la plus vaste unité de l'organisation politico-familiale des Mélanésiens de Fidji (aussi dits itaukei, terme qui se traduit par «maître » ou "propriétaire » de la terre). Le statut individuel s'acquiert le plus souvent en ligne paternelle, mais des variantes matrilinéaires et d'autres concernant la hiérarchie et les dénominations des unités familiales ont été décrites à Fidji.

8. Littéralement «la terre». Territoire traditionnel dominé par une chefferie, incluant l'espace maritime adjacent. Le terme vanua incorpore à la fois l'espace physique et la population qui y vit (« gens de la terre »).

9. À Fidji, le système de tenure maritime repose sur l'existence de 410 qoliqoli (territoires de pêche côtiers, allant en général jusqu'au bord externe du récif), de tailles variables, placés sous le contrôle des chefs de la population itaukei (mélanésienne). Un qoliqoli peut inclure des vasières, un espace de mangrove et des plages, un récif frangeant, ou encore un lagon et un récif barrière. Les membres d'une yavusa enregistrée comme rattachée à un qoliqoli donné disposent de droits d'usage exclusifs, sans être légalement considérés comme des "propriétaires » du domaine marin - ce qui est une différence fondamentale avec les règles régissant la propriété des native lands. L'exploitation commerciale des ressources marines est soumise à l'acquisition d'un permis, y compris pour les populations possédant les terres adjacentes, bien que cette obligation légale ne soit pas toujours respectée (Sloan \& Chand $2015: 1-2,6-7,23$ ).

10. Waya est connue dans la littérature pour les particularités de sa langue rattachée au « fidjien occidental ", interprétées comme étant le résultat d'une longue occupation humaine, peut-être même ininterrompue depuis l'époque Lapita (Pietrusewsky et al. 1997 et Gardner \& Pawley 2006 : 97). Au contraire de Waya, Matacawalevu n'a fait l'objet que de très peu de recherches en sciences humaines et sociales. Notons néanmoins que S. Best et G. Irwin ont inventorié sur cette île 18 sites archéologiques, dont certains ont livré de la céramique décorée (Cochrane $2004: 171$ ). 11. À Vuake, parmi les poissons les plus consommés figurent les perroquets, ulavi (Scarus spp.), les empereurs saint-pierre, kabatia (Lethrinus harak), les kanace (terme qui se rapporte à 
plusieurs espèces de Mugilidae), les chirurgiens striés, dridri (Ctenochaetus striatus). Des gastéropodes et mollusques bivalves, comme les arches kaikoso (Anadara spp.), des algues, des pieuvres harponnées sur le récif et des crustacés comme les crabes terrestres tuba (Cardisoma carnifex), complètent la diète quotidienne.

12. Les recherches d'A. Pawley (2011: 265, note 3) ont montré l'existence de près de 450 termes pour les poissons et 230 pour les invertébrés marins dans la langue de Waya, ce qui représente près de $85 \%$ des espèces animales nommées sur cette île.

13. Bau est le nom d'une île située près de la côte orientale de Viti Levu, dont les chefs ont longtemps exercé une position politique dominante à Fidji.

14. Ainsi, les personnes converties à l'Église Adventiste du Septième Jour s'abstiennent de consommer, et même de toucher tous les invertébrés marins, ainsi que les poissons sans écailles et nageoires, considérés comme impurs.

15. En 2012 une pêcheuse de Vuake pouvait obtenir jusqu'à $80 \mathrm{FJ} \$$ pour quatre qari vendus à un resort. Un pêcheur disposant d'un bateau pouvait pour sa part pêcher jusqu'à 300 à $400 \mathrm{FJ} \$$ de poisson en une seule nuit. Les salaires perçus dans les resorts étaient en moyenne de 50 à $100 \mathrm{FJ} \$$ pour une semaine, selon les tâches et responsabilités.

16. Comme le formulait un insulaire : "Work in the resort, you've got a boss there. And fishing, you're the boss. [...] In the resort you get stressed every time: 'you do that, you do that, you do this...' [...] You stay in the village, it's just up to you. You want to go fishing, you go. If you don't want to go, it's up to you » (A., 45 ans, Vuake).

17. En 2012, un bateau adapté à la pêche en haute-mer et son moteur étaient facturés environ $15000 \mathrm{FJ}$, une somme considérable dans ces communautés rurales.

18. C. Toren (1999 : 38, 43-44) a parlé pour sa part d'une purification de l'argent dans le contexte du rituel du gunu sede, pratiqué sur l'île de Gau, qui implique l'achat de coupes de yaqona, ou kava. Sur les tensions perçues entre certaines formes culturelles traditionnelles (coutumes d'emprunt, redistribution, philosophie collectiviste) et l'entreprenariat à Fidji, voir Farrelly \& Talatoka Vudiniabola 2013: 7-11. Sur le maintien des obligations de réciprocité voir aussi Williksen-Bakker 2002 et Gibson 2012 : 112, 114.

19. Tout pêcheur souhaitant pêcher dans un qoliqoli sur lequel il ne possède pas de droits traditionnels doit acquérir un permis signé par les autorités coutumières et validé par les autorités provinciales et le Département des Pêches du Commissioner Western Office, à Lautoka (Sloan \& Chand $2015: 6-7$ ).

20. Sur l'engouement pour les projets de conservation associant les communautés locales, leur diversité et leurs limites, voir notamment Hackel 1999 et Adams \& Hulme 2001.

21. Cette appellation a émergé en 2000, à l'issue d'une série de rencontres internationales dédiées à la conservation marine organisées à Fidji, aux Philippines et en Indonésie. H. Govan et al. (2008 : 2) donnent la définition suivante dans un guide du FLMMA Network destiné à faciliter la mise en place d'une AMGL: "An area of nearshore waters and coastal resources that is largely or wholly managed at a local level by the coastal communities, land-owning groups, partner organizations, and/or collaborative government representatives who reside or are based in the immediate area ». Ce texte invite à distinguer l'AMGL de l'« aire marine protégée » (marine protected area) vue comme typiquement associée à une approche "descendante " (top-down) de la conservation, même si certaines zones de l'AMGL peuvent être entièrement fermées à la pêche (ibid : 3 ).

22. Voir par exemple: "The Ucunivanua project set aside the usual mind-set that only experts know best and that development occurs only when planned by governments » (Aalbersberg et al. $2005: 144$ ). 


\section{RÉSUMÉS}

L'archipel des Yasawa est récemment devenu l'un des haut-lieux du tourisme à Fidji et le visage international de ce petit pays d'Océanie. Cet article examine la manière dont deux ensembles de phénomènes en partie liés - le développement du tourisme et celui d'une préoccupation environnementale suscitée par l'essor économique du pays et le changement climatique contribuent à y transformer les relations au monde marin, à partir de recherches conduites dans les villages de Yalobi et Vuake. Nous montrons que le tourisme accélère une certaine érosion du savoir coutumier relatif à la mer, tout en engendrant de nouveaux modes d'approche et d'usage du milieu marin - avec en particulier un essor de la pêche commerciale, qui permet de réguler ou compenser certaines conséquences du tourisme. Il apparaît d'autre part que pour les villageois de Yalobi et Vuake, la préservation du milieu marin ne saurait seulement passer par des dispositifs restrictifs, comme les tabous de pêche, que les Fidjiens sont actuellement invités à «redécouvrir». Elle implique aussi des actions positives de l'État pour protéger les droits traditionnels d'exploitation de la mer, ainsi que le maintien de relations harmonieuses à la fois avec les entités spirituelles, qui tirent les fils de l'existence des hommes, et au sein des communautés insulaires.

The Yasawa archipelago has recently become a major tourist hotspot in Fiji and the international face of this small country of Oceania. This paper examines how two related sets of phenomena tourism, and a growing concern over environmental issues due to economic development and climate change - contribute to re-shape relationships to the marine environment, relying on field research conducted in Yalobi and Vuake villages. It shows that tourism accelerates a loss of traditional marine knowledge, while leading to new approaches and uses of marine space and resources. For instance, commercial fishing, which helps regulate or compensate tourism impacts, is gaining importance. It is also concluded that, for Yalobi and Vuake villagers, preserving marine life cannot only rely on restrictive measures, such as fishing taboos, which Fijians are currently invited to "rediscover". It also involves positive actions from the State so as to protect traditional fishing rights and the preservation of harmonious relationships with spiritual entities, which pull the strings of human lives, and within the island community itself.

\section{INDEX}

Keywords : Oceania, tourism, marine resources, fishing, marine protected areas, participatory conservation

Index géographique : Fidji, Océanie

Mots-clés : tourisme, ressources marines, pêche, aires marines protégées, conservation participative 


\section{AUTEUR}

\section{ÉMILIE NOLET}

Maître de Conférences en Ethnoarchéologie de l'Océanie à l'Université Paris 1, PanthéonSorbonne, UMR 7041 ArScan. Institut d'Art et d'Archéologie, 3 rue Michelet, 75006 Paris.

Emilie.Nolet@univ-paris1.fr 